\title{
On the number of partition weights with Kostka multiplicity one
}

\author{
Zachary Gates* \\ Department of Mathematics \\ University of Virginia \\ Charlottesville, VA \\ zg8bf@virginia.edu \\ Brian Goldman ${ }^{\dagger}$ \\ Mathematics Department \\ College of William and Mary \\ Williamsburg, VA \\ bjgoldman@email.wm.edu \\ C. Ryan Vinroot ${ }^{\ddagger}$ \\ Mathematics Department \\ College of William and Mary \\ Williamsburg, VA \\ vinroot@math.wm.edu
}

Submitted: Jul 24, 2012; Accepted: Dec 19, 2012; Published: Dec 31, 2012

Mathematics Subject Classification: 05A19

\begin{abstract}
Given a positive integer $n$, and partitions $\lambda$ and $\mu$ of $n$, let $K_{\lambda \mu}$ denote the Kostka number, which is the number of semistandard Young tableaux of shape $\lambda$ and weight $\mu$. Let $J(\lambda)$ denote the number of $\mu$ such that $K_{\lambda \mu}=1$. By applying a result of Berenshtein and Zelevinskii, we obtain a formula for $J(\lambda)$ in terms of restricted partition functions, which is recursive in the number of distinct part sizes of $\lambda$. We use this to classify all partitions $\lambda$ such that $J(\lambda)=1$ and all $\lambda$ such that $J(\lambda)=2$. We then consider signed tableaux, where a semistandard signed tableau of shape $\lambda$ has entries from the ordered set $\{0<\overline{1}<1<\overline{2}<2<\cdots\}$, and such that $i$ and $\bar{i}$ contribute equally to the weight. For a weight $\left(w_{0}, \mu\right)$ with $\mu$ a partition, the signed Kostka number $K_{\lambda,\left(w_{0}, \mu\right)}^{ \pm}$is defined as the number of semistandard signed tableaux of shape $\lambda$ and weight $\left(w_{0}, \mu\right)$, and $J^{ \pm}(\lambda)$ is then defined to be the number of weights $\left(w_{0}, \mu\right)$ such that $K_{\lambda,\left(w_{0}, \mu\right)}^{ \pm}=1$. Using different methods than in the unsigned case, we find that the only nonzero value which $J^{ \pm}(\lambda)$ can take is 1 , and we find all sequences of partitions with this property. We conclude with an application of these results on signed tableaux to the character theory of finite unitary groups.
\end{abstract}

*Supported by NSF grant DMS-0854849.

†Supported by the College of William and Mary.

${ }^{\ddagger}$ Supported by NSF grant DMS-0854849. 


\section{Introduction}

Given partitions $\lambda$ and $\mu$ of a positive integer $n$, the Kostka number $K_{\lambda \mu}$ is the number of semistandard Young tableaux of shape $\lambda$ and weight $\mu$. Motivated by the importance of Kostka numbers and their generalizations in the representation theory of Lie algebras, Berenshtein and Zelevinskii [1] addressed the question of precisely when it is that $K_{\lambda \mu}=1$. It may be seen, for example, that we always $K_{\lambda \lambda}=1$. The results of this paper were motivated by the question: For which partitions $\lambda$ is it true that $K_{\lambda \mu}=1$ if and only if $\mu=\lambda$ ? The answer to this question, given in Corollary 5.1, turns out to be quite nice, which is that this holds exactly for those partitions $\lambda$ for which the conjugate partition $\lambda^{\prime}$ has distinct parts. In fact, using the result of Berenshtein and Zelevinskii, stated in Theorem 3.1 below, we can give much more information.

After giving some preliminary results and definitions for partitions in Section 2, and for tableaux in Section 3, we define $J(\lambda)$ to be the number of partitions $\mu$ such that $K_{\lambda \mu}=1$. Our first main result, given in Proposition 4.1 and Theorem 4.1, is a formula for $J(\lambda)$ in terms of restricted partition functions, as a recursive formula in terms of the number of distinct parts of $\lambda$. Using this formula, we are then able to give, for certain forms of $\lambda$, a product formula for $J(\lambda)$. We can apply this machinery quite effectively to obtain a classification of those $\lambda$ such that $J(\lambda)=1$ (Corollary 5.1), and those $\lambda$ such that $J(\lambda)=2$ (Corollary 5.2), as well as a characterization of those $\lambda$ such that $J(\lambda)$ is prime (Corollary 5.3).

In Section 6, we use completely different methods to analyze a similar question concerning the signed Kostka number. Given a partition $\lambda$, a semistandard signed tableau of shape $\lambda$ has entries from the ordered set $\{0<\overline{1}<1<\overline{2}<2<\cdots\}$, where entry $w_{i}$ of the weight is given by the total number of $i$ 's or $\bar{i}$ 's. Signed tableaux are a variation of symplectic tableaux, which were introduced by King [3] to describe the representations of $\operatorname{S~} p(2 n, \mathbb{C})$. Signed tableaux as we use here were applied in [5] to describe multiplicites in Gelfand-Graev characters of the finite unitary groups. Given a partition $\lambda$ and a weight $\left(w_{0}, \mu\right)$, where $\mu=\left(w_{1}, w_{2}, \ldots\right)$ is a partition, we define the signed Kostka number

$K_{\lambda,\left(w_{0}, \mu\right)}^{ \pm}$as the number of signed tableaux of shape $\lambda$ and weight $\left(w_{0}, \mu\right)$. We then define $J^{ \pm}(\lambda)$, given $\lambda$, as the number of weights $\left(w_{0}, \mu\right)$, where $\mu$ is a partition, such that the signed Kostka number $K_{\lambda,\left(w_{0}, \mu\right)}^{ \pm}=1$. In contrast with the question for unsigned tableaux, we find that even for multipartitions (or sequences of partitions) $\boldsymbol{\lambda}$, the only nonzero value $J^{ \pm}(\boldsymbol{\lambda})$ can take is 1 . We prove this statement, and classify all multipartitions $\boldsymbol{\lambda}$ such that $J^{ \pm}(\boldsymbol{\lambda})=1$, in Theorems 6.1 and 6.2. Finally, we give an application to the characters of the finite unitary group in Corollary 6.2, where we give a set of characters of the finite unitary group which occur with multiplicity 1 in a unique degenerate Gelfand-Graev character.

\section{Partitions and restricted partitions}

A partition of a non-negative integer $n$, written $\lambda \vdash n$, is a tuple of non-negative integers $\lambda=\left(\lambda_{1}, \lambda_{2}, \ldots, \lambda_{r}\right)$ such that $\lambda_{1} \geqslant \lambda_{2} \geqslant \cdots \geqslant \lambda_{r}$ and $\sum_{i=1}^{r} \lambda_{i}=n$. The size of $\lambda$ 
is then $n$, written $|\lambda|=n$. The numbers $\lambda_{i}$ are the parts of the partition, and the length of the partition, denoted $\ell(\lambda)$, is the number of positive parts of $\lambda$. While we allow parts of a partition to be 0 for some convenience, we consider two partitions to be identical if there is no difference in their positive parts. For a non-negative integer $n$, the partition function $p(n)$ is defined to be the number of distinct partitions of $n$ (note that $p(0)=1)$. Another notation for a partition that we will use is $\lambda=\left(k_{1}^{m_{1}}, k_{2}^{m_{2}}, \ldots, k_{s}^{m_{s}}\right)$, which denotes the partition with parts $\lambda_{i}=k_{1}$ for $1 \leqslant i \leqslant m_{1}$, and for $1<j \leqslant s, \lambda_{i}=k_{j}$ for $m_{j-1}+1 \leqslant i \leqslant m_{j-1}+m_{j}$. For example, the partition $\lambda=(7,7,7,5,5,3,2,2,2,2,1,1)$ will be denoted $\lambda=\left(7^{3}, 5^{2}, 3^{1}, 2^{4}, 1^{2}\right)$ in this notation.

Given a partition $\lambda=\left(\lambda_{1}, \lambda_{2}, \ldots, \lambda_{l}\right)$, the Young diagram for $\lambda$ is an array of leftjustified rows of boxes, where the $i$ th row from the top has $\lambda_{i}$ boxes. The conjugate of the partition of $\lambda$, denoted $\lambda^{\prime}$, is obtained by transposing the Young diagram of $\lambda$, changing rows into columns, and vice versa. For example, the Young diagram for $\lambda=(4,3,3,2,1)$ is

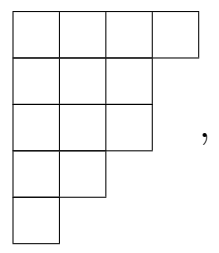

from which we see that $\lambda^{\prime}=(5,4,3,1)$, with Young diagram

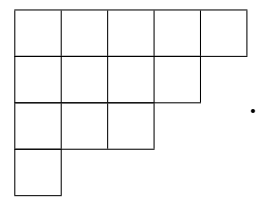

The dominance partial order on partitions of some fixed non-negative integer $n$, written $\unrhd$, is defined as follows. If $\lambda, \mu \vdash n$, then $\lambda \unrhd \mu$ if, for all $m \geqslant 1$, we have $\sum_{i=1}^{m} \lambda_{i} \geqslant \sum_{i=1}^{m} \mu_{i}$. For example, if $\lambda=(3,2,1), \mu=(3,1,1,1)$, and $\nu=(2,2,2)$, then $\lambda \unrhd \mu$ and $\lambda \unrhd \nu$, while $\mu$ and $\nu$ are incomparable.

Define $p_{l}(n)$ to be the number of partitions $\lambda$ of $n$ such that $\ell(\lambda) \leqslant l$, and define $p(k, l, n)$ to be the number of partitions $\lambda$ of $n$ such that $\lambda_{1} \leqslant k$ and $\ell(\lambda) \leqslant l$. In particular, note that if $l \geqslant n$, then $p_{l}(n)=p(n)$. Also, $p(n-1, l, n)=p_{l}(n)-1$. For any $n<0$, we define $p(k, l, n)=p_{l}(n)=p(n)=0$. We now make some observations which will be used crucially in the results in Section 4. We give an example with Young diagrams of the bijection in each proof below to demonstrate the straightforward arguments.

Lemma 2.1. For any positive integers $a, b$, and $n$, we have

$$
p(a, b, a b-n)=p(a, b, n) .
$$

In particular, if $a, b \geqslant n$, then $p(a, b, a b-n)=p(n)$.

Proof. Let $\lambda \vdash a b-n$ such that $\lambda_{1} \leqslant a$ and $\ell(\lambda) \leqslant b$, where $\lambda=\left(\lambda_{1}, \ldots, \lambda_{b}\right)$. The map $\lambda \mapsto \mu$, where $\mu$ is defined by $\mu_{i}=a-\lambda_{b-i+1}$ for $1 \leqslant i \leqslant b$, is a bijection from partitions $\lambda$ of $a b-n$ with $\lambda_{1} \leqslant a$ and $\ell(\lambda) \leqslant b$ to partitions $\mu$ of $n$ such that $\mu_{1} \leqslant a$ and 
$\ell(\mu) \leqslant b$. Note that the second statement follows from the first, since if $a, b \geqslant n$, then $p(a, b, n)=p(n)$.

If $a=5, b=6$, and $n=18$, then in the proof above, $\lambda=(4,4,2,1,1)$ maps to $\mu=(5,4,4,3,1,1)$, as follows:
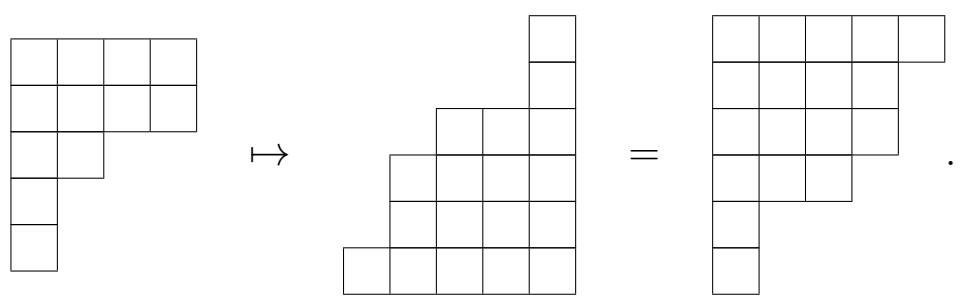

Lemma 2.2. Let $m, k \geqslant 1$. The number of partitions $\mu \vdash m k$ such that $\ell(\mu)=m+1$ and $\mu_{1} \leqslant k$ is $p_{m+1}(k)-1$.

Proof. Let $\mu$ be a partition of $m k$ with $\ell(\mu)=m+1$ and $\mu_{1} \leqslant k$. The map $\mu \mapsto \nu$, where $\nu$ is defined by $\nu_{i}=\mu_{i}-1$ for $1 \leqslant i \leqslant m+1$, defines a bijection from the set of partitions $\mu$ of $m k$ with $\ell(\mu)=m+1$ and $\mu_{1} \leqslant k$ to the partitions of $\nu$ of $m k-m-1$ with $\ell(\nu) \leqslant m+1$ and $\nu_{1} \leqslant k-1$. Thus, the number of such partitions $\mu$ is equal to $p(k-1, m+1, m k-m-1)$. By Lemma 2.1 with $a=k-1, b=m+1$ and $n=k$, we have $p(k-1, m+1, m k-m-1)=p(k-1, m+1, k)$. Since $p(k-1, m+1, k)=p_{m+1}(k)-1$, the result follows.

That is, in the proof of Lemma 2.2, we subtract 1 from each part of $\mu$, and then apply the bijection from Lemma 2.1 with $a=k-1, b=m+1$ and $n=k$. For example, if $m=5, k=3$, and $\mu=(3,3,3,3,2,1)$, then the resulting partition of $k=3$ after applying these maps is as follows, where we put dots in boxes which are removed in the subsequent map:

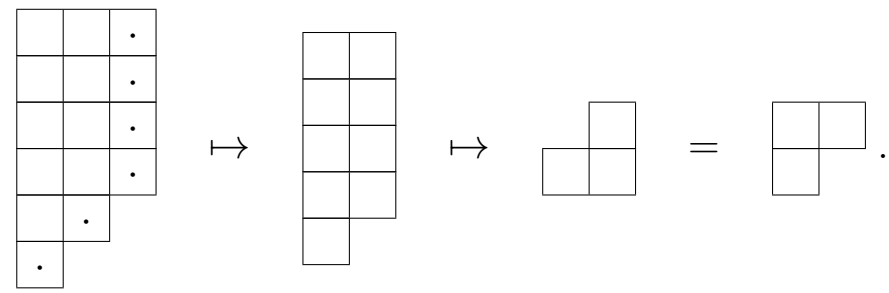

Lemma 2.3. Let $\lambda=\left(k_{1}^{m_{1}}, k_{2}\right)$, with $k_{1}>k_{2}>0$. The number of partitions $\mu$ of $m_{1} k_{1}+k_{2}$ such that $\ell(\mu)=m_{1}+1, \lambda \unrhd \mu$, and $\mu_{m_{1}+1} \neq k_{2}$ is $p_{m_{1}+1}\left(k_{1}-k_{2}\right)-1$.

Proof. First, we claim that if $\mu \vdash\left(m_{1} k_{1}+k_{2}\right)$ and $\ell(\mu)=m_{1}+1$, then $\lambda \unrhd \mu$ if and only if $\mu_{1} \leqslant k_{1}$. If $\mu_{1}>k_{1}=\lambda_{1}$, then it does not hold that $\lambda \unrhd \mu$. Conversely, suppose $\mu_{1} \leqslant k_{1}$. Then $\mu_{i} \leqslant k_{1}$ for $1 \leqslant i \leqslant m_{1}$, and so for any $m \leqslant m_{1}, \sum_{i=1}^{m} \mu_{i} \leqslant m k_{1}=\sum_{i=1}^{m} \lambda_{i}$. Thus $\lambda \unrhd \mu$.

Now note that if $\mu \vdash\left(m_{1} k_{1}+k_{2}\right), \ell(\mu)=m_{1}+1$ and $\mu_{1} \leqslant k_{1}$, then $\mu_{m_{1}+1} \geqslant k_{2}$. So, the partitions $\mu$ of $m_{1} k_{1}+k_{2}$ such that $\ell(\mu)=m_{1}+1, \lambda \unrhd \mu$, and $\mu_{m_{1}+1} \neq k_{2}$ are exactly those $\mu \vdash\left(m_{1} k_{1}+k_{2}\right)$ such that $\ell(\mu)=m_{1}+1, \mu \leqslant k_{1}$, and $\mu_{m_{1}+1} \geqslant k_{2}+1$. Given such a 
partition $\mu$, the map $\mu \mapsto \nu$, where $\nu$ is defined by $\nu_{i}=\mu_{i}-k_{2}-1$ for $i \leqslant m_{1}+1$, gives a bijection from partitions $\mu$ of $m_{1} k_{1}+k_{2}$ such that $\ell(\mu)=m_{1}+1, \lambda \unrhd \mu$, and $\mu_{m_{1}+1} \neq k_{2}$ to partitions $\nu$ of $m_{1} k_{1}+k_{2}-\left(m_{1}+1\right)\left(k_{2}+1\right)$ such that $\ell(\nu) \leqslant m_{1}+1$ and $\nu_{1} \leqslant k_{1}-k_{2}-1$. That is, the number of such partitions $\mu$ is exactly $p\left(k_{1}-k_{2}-1, m_{1}+1, m_{1} k_{1}-m_{1} k_{2}+m_{1}+1\right)$. By Lemma 2.1 with $a=k_{1}-k_{2}-1, b=m_{1}+1$, and $n=k_{1}-k_{2}$, this is equal to $p\left(k_{1}-k_{2}-1, m_{1}+1, k_{1}-k_{2}\right)=p_{m_{1}+1}\left(k_{1}-k_{2}\right)-1$.

That is, given $\mu$ as in the above proof, we subtract $k_{2}+1$ from each part of $\mu$, and then apply the bijection from Lemma 2.1 to obtain a restricted partition of $k_{1}-k_{2}$. For example, taking $\lambda=\left(6^{3}, 2\right)$, so $k_{1}=6, k_{2}=2$, and $m_{1}=3$, let $\mu=(6,5,5,4)$. Then, as shown below, the first map gives $\nu=(3,2,2,1)$, and applying the bijection from Lemma 2.1 with $a=3, b=4$, and $n=4$, yields the partition $(2,1,1)$ of $k_{1}-k_{2}=4$ :
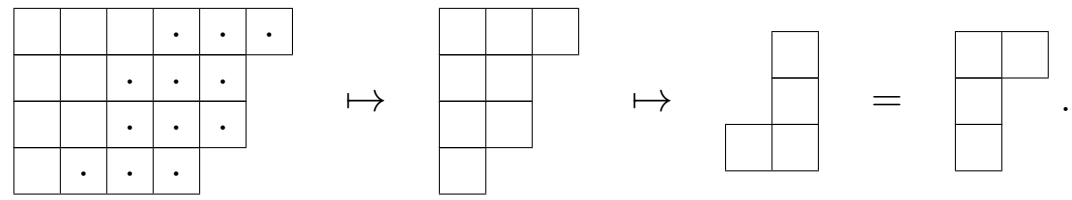

Lemma 2.4. Let $\lambda=\left(k_{1}, k_{2}^{j}\right)$, with $k_{1}>k_{2}>0$. The number of partitions $\mu$ of $k_{1}+j k_{2}$ such that $\ell(\mu)=j+1, \lambda \unrhd \mu$, and $\mu_{j+1} \neq k_{2}$ is $p_{j+1}\left(k_{1}-k_{2}-1-j\right)$.

Proof. We claim that if $\mu \vdash k_{1}+j k_{2}$ and $\ell(\mu)=j+1$, then $\lambda \unrhd \mu$ if and only if $\mu_{j+1} \geqslant k_{2}$. If $\mu_{j+1}<k_{2}$, then $\sum_{i=1}^{j} \mu_{i}=k_{1}+j k_{2}-\mu_{j+1}>k_{1}+(j-1) k_{2}=\sum_{i=1}^{j} \lambda_{i}$, and thus we cannot have $\lambda \unrhd \mu$. Conversely, suppose that $\mu_{j+1} \geqslant k_{2}$. Then $\mu_{i} \geqslant k_{2}$ for each $i \leqslant j+1$. It follows that $\lambda_{1}=k_{1} \geqslant \mu_{1}$, since $\sum_{i=2}^{j+1} \mu_{i} \geqslant j k_{2}$. Also, for any $1<m \leqslant j+1$, we have $\sum_{i=1}^{m} \lambda_{i}=k_{1}+(m-1) k_{2} \geqslant \sum_{i=1}^{m} \mu_{i}$, otherwise we would have $\sum_{i=1}^{j+1} \mu_{i}>k_{1}+j k_{2}$, since $\mu_{i} \geqslant k_{2}$ for each $i \leqslant j+1$. Thus $\lambda \unrhd \mu$ and the claim is proved.

We have from the claim that the set of partitions $\mu$ of $k_{1}+j k_{2}$ such that $\ell(\mu)=j+1$, $\lambda \unrhd \mu$, and $\mu_{j+1} \neq k_{2}$ is exactly the set of partitions $\mu$ of $k_{1}+j k_{2}$ with $\ell(\mu)=j+1$ and $\mu_{j+1} \geqslant k_{2}+1$. Given such a partition $\mu$, define a partition $\nu$ by $\nu_{i}=\mu_{i}-k_{2}-1$ for $i=1, \ldots, j+1$, which results in a partition of $k_{1}+j k_{2}-(j+1)\left(k_{2}+1\right)=k_{1}-k_{2}-1-j$ of length at most $j+1$. Moreover, the map $\mu \mapsto \nu$ provides a bijection from partitions $\mu$ of $k_{1}+j k_{2}$ with $\ell(\mu)=j+1, \lambda \unrhd \mu$, and $\mu_{j+1} \neq k_{2}$ to partitions $\nu$ of $k_{1}-k_{2}-1-j$ with length at most $j+1$, giving the desired enumeration.

To demonstrate the proof of Lemma 2.4 , suppose $\lambda=\left(13,1^{2}\right)$, so $k_{1}=13, k_{2}=1$, and $j=2$, and suppose $\mu=(6,5,4)$, so $\ell(\mu)=j+1$ and $\mu_{j+1}=4 \geqslant k_{2}+1$. The map sends $\mu$ to $\nu=(4,3,2)$, as shown below:

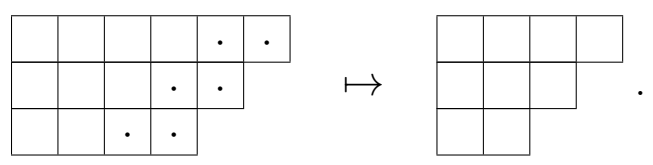




\section{Tableaux and Kostka multiplicity}

A semistandard Young tableau of shape $\lambda$, is a filling of the boxes of the Young diagram for $\lambda$ with positive integers in such a way that rows are non-decreasing from left to right, and columns are increasing from top to bottom. The weight of a semistandard Young tableau is a tuple $\alpha=\left(\alpha_{1}, \alpha_{2}, \ldots\right)$, where $\alpha_{i}$ is the number of $i$ 's which appear in the tableau. Note that when $\alpha$ is the weight of a tableau of shape $\lambda$, where $\lambda \vdash n$, then $\sum_{i} \alpha_{i}=n$, that is, $\alpha$ is a composition of $n$.

Example. Let $\lambda=(5,4,2,1,1)$. Then the following are two different semistandard Young tableaux of shape $\lambda$ and weight $(1,4,2,3,0,1,1)$ :

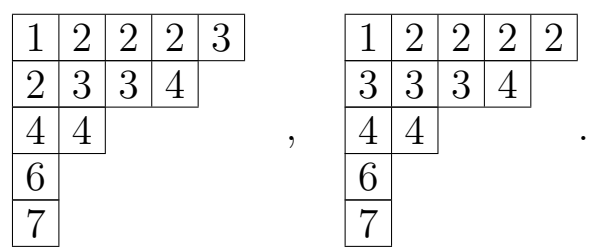

Since all of our tableaux will be semistandard Young tableaux, we will henceforth refer to them as simply Young tableaux.

Given a partition $\lambda$ of $n$ and a composition $\alpha$ of $n$, the Kostka number, denote $K_{\lambda \alpha}$, is defined to be the number of distinct Young tableaux of shape $\lambda$ and weight $\alpha$. It is known that the value of $K_{\lambda \alpha}$ is invariant under permutation action on the parts of $\alpha$, and so we may assume that $\alpha$ is a partition of $n$. Furthermore, it is known that if $\lambda, \mu \vdash n$, then $K_{\lambda \mu} \neq 0$ if and only if $\lambda \unrhd \mu$ (where $\unrhd$ is the dominance partial ordering).

As mentioned in the introduction, Kostka numbers play an important role in representation theory and algebraic combinatorics. If $s_{\lambda}$ denotes the Schur symmetric function, and $h_{\mu}$ denotes the complete symmetric function, then the Kostka numbers appear in the transition matrix for these bases (as well as being involved in many other transition matrices for bases, see [4, Chapter I.6, Table 1]):

$$
h_{\mu}=\sum_{\lambda \unrhd \mu} K_{\lambda \mu} s_{\lambda}
$$

This fact is essentially equivalent to the following statement regarding representations of the symmetric group. If $\lambda, \mu \vdash n$, let $S_{\mu}$ denote the Young subgroup corresponding to $\mu$ of the symmetric group $S_{n}$, let $\mathbf{1}$ denote the trivial representation, and let $\pi_{\lambda}$ denote the complex irreducible representation of $S_{n}$ indexed by $\lambda$. Then (see [2, Corollary 4.9]):

$$
\operatorname{Ind}_{S_{\mu}}^{S_{n}}(\mathbf{1}) \cong \bigoplus_{\lambda \unrhd \mu} K_{\lambda \mu} \pi_{\lambda}
$$

Kostka numbers and their variations also appear in the representation theory of Lie algebras, Weyl groups, and finite groups of Lie type, all as multiplicities in the decomposition of some representation (we will see an application of this to the finite unitary 
group). A representation having multiplicity one in the decomposition of another representation is an important property in representation theory for many reasons, such as the multiplicity one representation inheriting certain properties of others, it is an important question to ask precisely when a Kostka number is equal to 1. This was the motivation for Berenshtein and Zelevinskii [1, Theorem 1.5] to prove the following criterion for when $K_{\lambda \mu}=1$.

Theorem 3.1 (Berenshtein and Zelevinskii). Let $\lambda=\left(\lambda_{1}, \ldots, \lambda_{l}\right)$ and $\mu=\left(\mu_{1}, \ldots, \mu_{l}\right)$, where some $\lambda_{i}$ or $\mu_{i}$ may equal 0 (so there is some choice in l). Then $K_{\lambda \mu}=1$ if and only if there exists a choice of indices $0=i_{0}<i_{1}<\cdots<i_{k}=l$ such that, for $j=1, \ldots, k$, the sub-partitions $\lambda^{j}=\left(\lambda_{i_{j-1}+1}, \ldots, \lambda_{i_{j}}\right)$ and $\mu^{j}=\left(\mu_{i_{j-1}+1}, \ldots, \mu_{i_{j}}\right)$ satisfy the following:

1. $\left|\lambda^{j}\right|=\left|\mu^{j}\right|$ and $\lambda^{j} \unrhd \mu^{j}$, and

2. either $\lambda_{i_{j-1}+1}=\lambda_{i_{j-1}+2}=\cdots=\lambda_{i_{j}-1}$ or $\lambda_{i_{j-1}+2}=\cdots=\lambda_{i_{j}}$.

Example. While it is well-known that $K_{\lambda \lambda}=1$ for any partition $\lambda$, we may see that it follows from Theorem 3.1 by choosing $i_{j}=j$ for each $j$, so that $\lambda^{j}=\left(\lambda_{j}\right)=\mu^{j}$. Since this choice of indices immediately satisfies the conditions of the theorem, we have $K_{\lambda \lambda}=1$.

Example. Let $\lambda=(4,4,2,2,1)$ and $\mu=(4,3,3,2,1)$. Consider the indices $i_{1}=3$, $i_{2}=5$, so that $\lambda^{1}=(4,4,2), \mu^{1}=(4,3,3)$, and $\lambda^{2}=\mu^{2}=(2,1)$. Since these satisfy the conditions of Theorem 3.1, then $K_{\lambda \mu}=1$. In fact, we shall see in Corollary 5.2 that $\mu$ is the only partition other than $\lambda$ with this property. The unique tableau of shape $\lambda$ and weight $\mu$ is

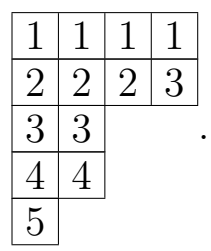

While Berenshtein and Zelevinskii give a certain enumeration of pairs $(\lambda, \mu)$ such that $K_{\lambda \mu}=1$ in terms of Lie theory and a reduction to "primitive pairs" [1, Theorem 1.3], it is not obvious how to obtain an explicit enumeration of, given a partition $\lambda$, all partitions $\mu$ such that $K_{\lambda \mu}=1$. We address this question in the next two sections. For this purpose, for any partition $\lambda \vdash n$, we define $J(\lambda)$ by

$$
J(\lambda)=\#\left\{\mu \vdash n \mid K_{\lambda \mu}=1\right\} .
$$

So, when $\lambda=(n)$, we have $J(\lambda)=p(n)$, and when $\lambda=\left(1^{n}\right)$, then $J(\lambda)=1$.

\section{A recursion for $J(\lambda)$}

We begin by evaluating $J(\lambda)$ when all parts of $\lambda$ are equal, that is, when $\lambda=\left(k^{m}\right)$ for some $m, k>0$. Somewhat surprisingly, if we fix $k$, then we find that $J\left(k^{m}\right)$ does not increase without bound with $m$, but rather reaches its maximum value for $m=k-1$, and is equal to this value for any $m \geqslant k-1$. 
Proposition 4.1. Let $\lambda=\left(k^{m}\right)$. Then

$$
J(\lambda)=p(k)+p_{m+1}(k)-1-\lfloor k / 2\rfloor=p(k)+p_{m+1}(k)-p_{2}(k) .
$$

In particular, if $m \geqslant k-1$, then $J(\lambda)=2 p(k)-1-\lfloor k / 2\rfloor=2 p(k)-p_{2}(k)$.

Proof. In applying Theorem 3.1, we must count how many distinct partitions $\mu \vdash k m$ are possible when choosing different indices which give sub-partitions $\lambda^{j}$ of $\lambda$. Recall that we may consider $\lambda$ as ending with any number of 0's as parts, which may allow for different choices of indices which satisfy the conditions of Theorem 3.1.

To better illustrate the counting argument, we consider the running example $\lambda=\left(4^{3}\right)$.

First, consider the case when $\lambda^{1}=\left(k^{m-1}\right)$, which corresponds to the choice $i_{1}=m-1$. Then, we are forced to also have $\mu^{1}=\left(k^{m-1}\right)$ in order to have $\lambda^{1} \unrhd \mu^{1}$. We must then have $\lambda^{2}=(k, 0, \ldots, 0)$, where we may have any number of 0 's appear. In order for $\lambda^{2} \unrhd \mu^{2}$, we only require $\left|\mu^{2}\right|=k$. Thus, the number of distinct choices for $\mu \vdash k m$ in the case $i_{1}=m-1$ is exactly $p(k)$, corresponding to the $p(k)$ partitions of $k m$ with first $m-1$ parts equal to $k$. In the example $\lambda=\left(4^{3}\right)$, the $\mu$ which we are counting correspond to the tableaux of shape $\lambda$ with 1's and 2's filling the first two rows, and the third row having $p(4)=5$ possible fillings. Corresponding to the partition $(2,1,1)$ of 4 is the following tableau of shape $\lambda$ and weight $(4,4,2,1,1)$ :

\begin{tabular}{|l|l|l|l|}
\hline 1 & 1 & 1 & 1 \\
\hline 2 & 2 & 2 & 2 \\
\hline 3 & 3 & 4 & 5 \\
\hline
\end{tabular}

If we consider $\lambda^{1}=\left(k^{m}\right)$, then we are forced to have $\mu^{1}=\left(k^{m}\right)$ as well, and so $\mu=\left(k^{m}\right)$, which is accounted for in the first case above, with $\mu^{1}=\left(k^{m-1}\right)$ and $\mu^{2}=(k)$. So instead, consider the case that $\lambda^{1}=\left(k^{m}, 0\right)$. Since the case for $\mu=\left(k^{m}\right)$ is already counted, we do not consider $\mu^{1}=\left(k^{m}, 0\right)$. In order for $\lambda^{1} \unrhd \mu^{1}$, we need $\mu_{1}^{1} \leqslant k$, and for the conditions on the indices to be met, we also need $\ell\left(\mu^{1}\right)=m+1$. Once this choice for $\mu^{1}$ is made, the partition $\mu$ is determined. By Lemma 2.2, the number of such $\mu^{1}$ is equal to $p_{m+1}(k)-1$. There is intersection, however, with this set of $\mu$, and those $\mu$ obtained in the case that $\lambda^{1}=\left(k^{m-1}\right)$. The $\mu$ that are in common with both cases are those such that $\ell(\mu)=m+1$, and $\mu_{i}=k$ for $1 \leqslant i \leqslant m-1$. In the case $\lambda^{1}=\left(k^{m-1}\right)$, these correspond to when $\ell\left(\mu^{2}\right)=2$, with $\mu^{2} \vdash k$. That is, we have overcounted by the number of partitions of $k$ with exactly 2 parts, of which there are $\lfloor k / 2\rfloor$. So, the total number of $\mu$ such that $K_{\lambda \mu}=1$ thus far is exactly $p(k)+p_{m+1}(k)-1-\lfloor k / 2\rfloor$. In the example $\lambda=\left(4^{3}\right)$, we are counting those $\mu$ with 4 parts and such that $\mu_{1} \leqslant 4$ and $\mu_{2} \neq 4$. The tableau corresponding to $\mu=\left(3^{4}\right)$ in this count is:

\begin{tabular}{|l|l|l|l|}
\hline 1 & 1 & 1 & 2 \\
\hline 2 & 2 & 3 & 3 \\
\hline 3 & 4 & 4 & 4 \\
\hline
\end{tabular}

Finally, consider when $\lambda^{1}=\left(k^{n_{1}}\right)$ for some $n_{1}<m-1$. Then for some $s>1$, we have $\lambda^{j}=\left(k^{n_{j}}\right)$ for $1 \leqslant j<s$, with either $\lambda^{s}=\left(k^{n_{s}}\right),\left(k^{n_{s}}, 0\right)$, or $(k, 0, \ldots, 0)$ (for any number 
of 0's, with $\left.n_{s}=1\right)$, where $n_{1}+\cdots+n_{s}=m$. This forces $\mu^{j}=\left(k^{n_{j}}\right)$ for $1 \leqslant j<s$, and $\mu^{s}$ to be a partition such that $\mu$ is one of the partitions already considered.

So, we have counted the total number of $\mu$ such that $K_{\lambda \mu}=1$, giving $J(\lambda)=p(k)+$ $p_{m+1}(k)-1-\lfloor k / 2\rfloor$, where $1+\lfloor k / 2\rfloor=p_{2}(k)$. The second statement follows from the first since $p_{m+1}(k)=p(k)$ when $m+1 \geqslant k$.

We now enumerate $J(\lambda)$ in the general case. In Theorem 4.1 below, along with Proposition 4.1, we obtain a method of calculating $J(\lambda)$ recursively, in terms of the number of distinct part sizes of the partition. That is, if we know $J(\lambda)$ whenever $\lambda$ has fewer than $s$ different part sizes, then we may compute $J(\lambda)$ whenever $\lambda$ has $s$ different part sizes. In the end, the expression for $J(\lambda)$ is in terms of restricted partition functions of numbers no larger than the difference of consecutive part sizes.

Theorem 4.1. Let $\lambda=\left(k_{1}^{m_{1}}, k_{2}^{m_{2}}, \ldots, k_{s}^{m_{s}}\right)$, with $s \geqslant 2$. Then

$$
\begin{aligned}
J(\lambda)= & J\left(k_{2}^{m_{2}}, \ldots, k_{s}^{m_{s}}\right)+\left(p_{m_{1}+1}\left(k_{1}-k_{2}\right)-1\right) J\left(k_{2}^{m_{2}-1}, k_{3}^{m_{3}}, \ldots, k_{s}^{m_{s}}\right) \\
& +\sum_{j=2}^{\min \left\{m_{2}, k_{1}-k_{2}-1\right\}} p_{j+1}\left(k_{1}-k_{2}-1-j\right) J\left(k_{2}^{m_{2}-j}, k_{3}^{m_{3}}, \ldots, k_{s}^{m_{s}}\right) .
\end{aligned}
$$

Proof. Like in the proof of Proposition 4.1, we apply Theorem 3.1 to count those $\mu$ such that $K_{\lambda \mu}=1$, by considering the various possibilities for the first sub-partition $\lambda^{1}$ of $\lambda$ chosen. As in the proof of Proposition 4.1, we will keep a running example, with $\lambda=\left(8,4^{3}, 2,1\right)$, to make the method of enumeration more clear.

First, consider the case when $\lambda^{1}=\left(k_{1}^{m_{1}}\right)$, in which case we are forced to choose $\mu^{1}=\left(k_{1}^{m_{1}}\right)$. Let $\tilde{\lambda}=\left(k_{2}^{m_{2}}, \ldots, k_{s}^{m_{s}}\right)$. We must now choose our partition $\mu$ as follows, in order to have $K_{\lambda \mu}=1$. Let $\tilde{\mu}$ denote the partition obtained by deleting the parts of $\mu^{1}$ from $\mu$. Then the conditions of Theorem 3.1 are satisfied if and only if $K_{\tilde{\lambda} \tilde{\mu}}=1$. The total number of such $\tilde{\mu}$, and so the total number of $\mu$ in this case, is thus $J(\tilde{\lambda})=J\left(k_{2}^{m_{2}}, \ldots, k_{s}^{m_{s}}\right)$. Included here are also those cases when $\lambda^{i}=\left(k_{1}^{n_{i}}\right)$ for some $n_{i}>0,1 \leqslant i \leqslant d$, such that $n_{1}+\cdots+n_{d}=m_{1}$, since we would then also be forced to choose $\mu$ such that its first $m_{1}$ parts are equal to $k_{1}$. With $\lambda=\left(8,4^{3}, 2,1\right)$, these correspond to the tableaux of the form

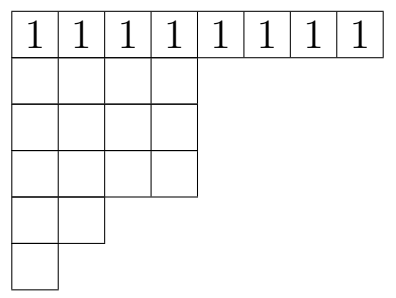

where the blank boxes may be filled in $J(\tilde{\lambda})$ ways, where $\tilde{\lambda}=\left(4^{3}, 2,1\right)$ here.

Now consider the case that $\lambda^{1}=\left(k_{1}^{m_{1}}, k_{2}\right)$. We must then choose $\mu^{1} \vdash\left(m_{1} k_{1}+k_{2}\right)$ such that $\ell\left(\mu^{1}\right)=m_{1}+1$ and $\lambda^{1} \unrhd \mu^{1}$. However, if $\mu_{m_{1}+1}^{1}=k_{2}$, then $\mu^{1}=\lambda^{1}$, and the resulting $\mu$ has $k_{1}$ as its first $m_{1}$ parts, which is counted in the first case above. Thus, we must the count the number of partitions of $\mu^{1}$ with the conditions already stated, and such 
that $\mu_{m_{1}+1}^{1} \neq k_{2}$. By Lemma 2.3 , the number of such $\mu^{1}$ is equal to $p_{m_{1}+1}\left(k_{1}-k_{2}\right)-1$, and recall from the proof of Lemma 2.3, this also implies $\mu_{m_{1}+1}^{1} \geqslant k_{2}+1$. If we now let $\tilde{\lambda}=\left(k_{2}^{m_{2}-1}, k_{3}^{m_{3}}, \ldots, k_{s}^{m_{s}}\right)$, and $\tilde{\mu}$ denote the partition obtained by deleting the first $m_{1}+1$ parts of a desired partition $\mu$, then we have $K_{\lambda \mu}=1$ if and only if $K_{\tilde{\lambda} \tilde{\mu}}=1$. That is, the total number of $\mu$ such that $K_{\lambda \mu}=1$ when $\lambda^{1}=\left(k_{1}^{m_{1}}, k_{2}\right)$ which were not counted in the first case is $\left(p_{m_{1}+1}\left(k_{1}-k_{2}\right)-1\right) J\left(k_{2}^{m_{2}-1}, k_{3}^{m_{3}}, \ldots, k_{s}^{m_{s}}\right)$. Included in the count for this case are all cases for which there are $n_{i}>0,1 \leqslant i \leqslant d$, with $n_{1}+\cdots+n_{d}=m_{1}$, where $\lambda^{i}=\left(k_{1}^{n_{i}}\right)$ for $i \leqslant d-1$ and $\lambda^{d}=\left(k_{1}^{n_{d}}, k_{2}\right)$. These are included in the $\mu^{1}$ just counted which have $k_{1}$ as their first $n_{1}+\cdots+n_{d-1}$ parts. In the running example of $\lambda=\left(8,4^{3}, 2,1\right)$, we are counting tableaux such that $\mu^{1}$ satisfies $\ell\left(\mu^{1}\right)=2$, with $\mu_{2}^{1} \geqslant 5$, and such that $(8,4) \unrhd \mu^{1}$, and there are $p_{2}(4)-1=2$ such $\mu^{1}$, which are $(7,5)$ and $(6,6)$. In this case, the tableaux enumerated are of the form

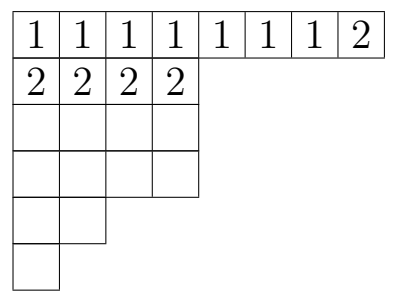

and

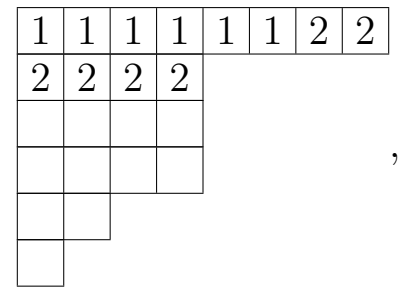

where the blank boxes may be filled in $J\left(4^{2}, 2,1\right)$ ways.

Finally, consider the case when there is a $d \geqslant 1$ and a $j \leqslant m_{2}$ such that $\lambda^{d}=\left(k_{1}, k_{2}^{j}\right)$. So that we do not count some of the previous cases twice, we restrict $j \geqslant 2$. Here, for each $i<d, \lambda^{i}$ has all parts equal to $k_{1}$, forcing $\mu^{i}$ for $i<d$ to be the same, and so the first $m_{1}-1$ parts of $\mu$ are equal to $k_{1}$. We must count the number of $\mu^{d}$ such that $\mu^{d} \vdash\left(k_{1}+j k_{2}\right)$, $\ell\left(\mu^{d}\right)=j+1$, and $\lambda^{d} \unrhd \mu^{d}$. As in the proof of Lemma 2.4, this implies $\mu_{j+1}^{d} \geqslant k_{2}$. If $\mu_{j+1}^{d}=k_{2}$, then the resulting $\mu$ can be obtained by considering $\lambda^{d}=\left(k_{1}, k_{2}^{j-1}\right)$. To prevent overcounting in this way, we must restrict $\mu_{j+1}^{d} \neq k_{2}$, so $\mu_{j+1}^{d}=\mu_{m_{1}+j}>k_{2}$. Furthermore, for any $l<j$, if $\lambda^{d}=\left(k_{1}, k_{2}^{l}\right)$, then we necessarily have $\mu_{m_{1}+j} \leqslant k_{2}$. That is, we are counting all new cases by counting the partitions $\mu^{d}$ of $k_{1}+k_{2} j$ with $\ell\left(\mu^{d}\right)=j+1$ and $\mu_{j+1}^{d} \neq k_{2}$. By Lemma 2.4, the number of such partitions $\mu^{d}$ is $p_{j+1}\left(k_{1}-k_{2}-1-j\right)$. Similar to the previous cases, the number of ways to choose the rest of the partition $\mu$ so that $K_{\lambda \mu}=1$ is $J\left(k_{2}^{m_{2}-j}, k_{3}^{m_{3}}, \ldots, k_{s}^{m_{s}}\right)$. So, for our fixed $j, 2 \leqslant j \leqslant m_{2}$, the total number of possible $\mu$ is $p_{j+1}\left(k_{1}-k_{2}-1-j\right) J\left(k_{2}^{m_{2}-j}, k_{3}^{m_{3}}, \ldots, k_{s}^{m_{s}}\right)$. Since this expression vanishes when $j>k_{1}-k_{2}-1$, we need only consider when $j \leqslant \min \left\{m_{2}, k_{1}-k_{2}-1\right\}$. In the case $\lambda=\left(8,4^{3}, 2,1\right)$, we have $m_{2}=k_{1}-k_{2}-1=3$, so we must consider $j=2,3$. For $j=2$, we have $\lambda^{1}=\left(8,4^{2}\right)$, and we must have $\ell\left(\mu^{1}\right)=3$ and $\lambda^{1} \unrhd \mu^{1}$, and $\mu_{3}^{1} \geqslant 5$. There is $p_{3}(1)=1$ such partition, which is $\mu^{1}=\left(6,5^{2}\right)$. The resulting tableaux are of the form

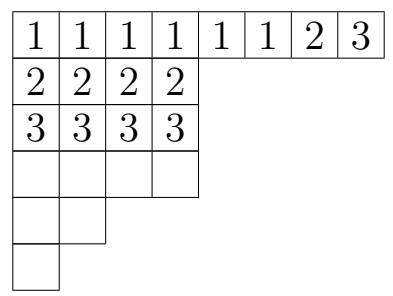


where the empty boxes may be filled in $J(4,2,1)$ ways. For $j=3, \lambda^{1}=\left(8,4^{3}\right)$, and $\mu^{1}$ must satisfy $\ell\left(\mu^{1}\right)=4, \lambda^{1} \unrhd \mu^{1}$, and $\mu_{4}^{1} \geqslant 5$. There is again only $p_{4}(0)=1$ such partition, given by $\mu^{1}=\left(5^{4}\right)$. The corresponding tableaux of shape $\lambda$ are of the form

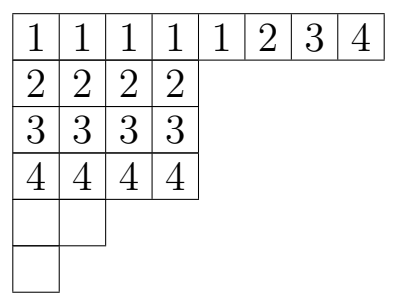

with $J(2,1)$ possible ways to fill in blank boxes.

We have now accounted for any $\mu$ arising from possible choices of sub-partitions of $\lambda$, and taking the sum of all of these cases gives the result.

We now obtain some interesting properties of $J(\lambda)$ which follow from Theorem 4.1.

Corollary 4.1. If $m_{1} \geqslant k_{1}-k_{2}-1$, then $J\left(k_{1}^{m_{1}}, k_{2}^{m_{2}}, \ldots, k_{s}^{m_{s}}\right)=J\left(k_{1}^{k_{1}-k_{2}-1}, k_{2}^{m_{2}}, \ldots, k_{s}^{m_{s}}\right)$. If $k_{1}-k_{2}=1$, then $J\left(k_{1}^{m_{1}}, k_{2}^{m_{2}}, \ldots, k_{s}^{m_{s}}\right)=J\left(k_{2}^{m_{2}}, \ldots, k_{s}^{m_{s}}\right)$.

Proof. If $m_{1} \geqslant k_{1}-k_{2}-1$, then $p_{m_{1}+1}\left(k_{1}-k_{2}\right)=p\left(k_{1}-k_{2}\right)=p_{k_{1}-k_{2}}\left(k_{1}-k_{2}\right)$, and when $k_{1}-k_{2}=1, p_{m_{1}+1}\left(k_{1}-k_{2}\right)-1=p_{j+1}\left(k_{1}-k_{2}-1-j\right)=0$, and so the results follows immediately from Theorem 4.1.

Corollary 4.2. Let $\lambda=\left(\lambda_{1}, \lambda_{2}, \ldots, \lambda_{\ell}\right)=\left(k_{1}^{m_{1}}, k_{2}^{m_{2}}, \ldots, k_{s}^{m_{s}}\right)$, and $\tilde{\lambda}=\left(\lambda_{i}, \lambda_{i+1}, \ldots, \lambda_{\ell}\right)$ for some $1 \leqslant i \leqslant \ell$. Then $J(\tilde{\lambda}) \leqslant J(\lambda)$.

Proof. It is enough to prove the statement for $i=2$. If $m_{1}=1$, then the first term in the recursive formula for $J(\lambda)$ in Theorem 4.1 is $J(\tilde{\lambda})$, and the result follows. Suppose $m_{1}>1$. If $k_{1}-k_{2}=1$, then by Corollary $4.1, J(\lambda)=J(\tilde{\lambda})=J\left(k_{2}^{m_{2}}, \ldots, k_{s}^{m_{s}}\right)$. If $k_{1}-k_{2}>1$, then the second term of the formula for $J(\lambda)$ in Theorem 4.1 is $\left(p_{m_{1}+1}\left(k_{1}-k_{2}\right)-1\right) J(\tilde{\lambda})$, where $p_{m_{1}+1}\left(k_{1}-k_{2}\right)>1$, and the result follows again.

When a partition $\lambda$ has distinct parts, then the formula in Theorem 4.1 simplifies to a particularly nice form, as follows.

Corollary 4.3. Let $\lambda=\left(k_{1}, k_{2}, \ldots, k_{s}\right)$ with $k_{1}>k_{2}>\cdots>k_{s}>0$, so $\lambda$ has distinct parts. Let $J_{i}=J\left(k_{s-i+1}, k_{s-i+2}, \ldots, k_{s}\right)$ for $1 \leqslant i \leqslant s$, and define $J_{0}=1, k_{0}=0$. Then, for $1<i \leqslant s, J_{i}=J_{i-1}+\left\lfloor\left(k_{i}-k_{i-1}\right) / 2\right\rfloor J_{i-2}$

Proof. Since $m_{i}=1$ for $1 \leqslant i \leqslant s$, then the third term in the equation for $J(\lambda)$ in Theorem 4.1 is an empty sum. Also, $p_{m_{i}+1}\left(k_{i}-k_{i-1}\right)-1=\left\lfloor\left(k_{i}-k_{i-1}\right) / 2\right\rfloor$, and the result follows from Theorem 4.1.

Example 1. If $m>1$ and $\lambda=(2 m-1,1)$, then $J(\lambda)=m$. In particular, $J(\lambda)$ may take any positive integer value. In fact, for any positive integers $m$ and $l \geqslant 2$, we may find a partition $\lambda$ such that $J(\lambda)=m$ and $\ell(\lambda)=l$, since by Corollary 4.1, $J\left(2 m^{l-2}, 2 m-1,1\right)=J(2 m-1,1)=m$. Or, if we would like such a partition with distinct 
parts, then also by Corollary 4.1 , if $\lambda=(2 m-1+l-2,2 m-1+l-3, \ldots, 2 m-1,1)$, then $J(\lambda)=m$.

Example 2. Consider $\lambda=(2 n-1,2 n-3, \ldots, 3,1)$. Then $k_{i}-k_{i-1}=2$ for each $i$, and $J_{1}=J(1)=1$. It follows from Corollary 4.3 that $J(\lambda)=F_{n+1}$, the $(n+1)$-st Fibonacci number.

\section{A product formula}

We now obtain a result that allows us to compute $J(\lambda)$ by decomposing $\lambda$, under certain conditions. In particular, $J(\lambda)$ may be written as a product of the $J$-values of smaller partitions under these conditions. The inequalities we obtain below without restrictions are also useful, in the corollaries which follow.

Theorem 5.1. Let $\lambda=\left(k_{1}^{m_{1}}, k_{2}^{m_{2}}, \ldots, k_{s}^{m_{s}}\right)$, with $s \geqslant 2$. Then for any $1<i \leqslant s$, we have

$$
J(\lambda) \geqslant J\left(\left(k_{1}-k_{i}+1\right)^{m_{1}},\left(k_{2}-k_{i}+1\right)^{m_{2}}, \ldots, 1^{m_{i}}\right),
$$

and

$$
J(\lambda) \leqslant J\left(\left(k_{1}-k_{i}+1\right)^{m_{1}},\left(k_{2}-k_{i}+1\right)^{m_{2}}, \ldots, 1^{m_{i}}\right) J\left(k_{i}^{m_{i}}, k_{i+1}^{m_{i+1}}, \ldots, k_{s}^{m_{s}}\right) .
$$

If either $k_{i-1}-k_{i}=1$ or $m_{i} \geqslant k_{i-1}-k_{i+1}-2$, then

$$
J(\lambda)=J\left(\left(k_{1}-k_{i}+1\right)^{m_{1}},\left(k_{2}-k_{i}+1\right)^{m_{2}}, \ldots, 1^{m_{i}}\right) J\left(k_{i}^{m_{i}}, k_{i+1}^{m_{i+1}}, \ldots, k_{s}^{m_{s}}\right) .
$$

Proof. Consider the statements for $i=2$. By Theorem 4.1, we have

$J\left(\left(k_{1}-k_{2}+1\right)^{m_{1}}, 1^{m_{2}}\right)=1+\left(p_{m_{1}+1}\left(k_{1}-k_{2}\right)-1\right)+\sum_{j=2}^{\min \left\{m_{2}, k_{1}-k_{2}-1\right\}} p_{j+1}\left(k_{1}-k_{2}-1-j\right)$,

since $J\left(1^{m_{2}}\right)=J\left(1^{m_{2}-j}\right)=1$ for any $j \leqslant m_{2}$. Since $J\left(k_{2}^{m_{2}-j}, k_{3}^{m_{3}}, \ldots, k_{s}^{m_{2}}\right) \geqslant 1$ for $0 \leqslant j \leqslant m_{2}$, it follows from the formula for $J(\lambda)$ in Theorem 4.1 that $J(\lambda) \geqslant J\left(\left(k_{1}-\right.\right.$ $\left.\left.k_{2}+1\right)^{m_{1}}, 1^{m_{2}}\right)$. Also,

$$
\begin{gathered}
J\left(\left(k_{1}-k_{2}+1\right)^{m_{1}}, 1^{m_{2}}\right) J\left(k_{2}^{m_{2}},\right. \\
\left.k_{3}^{m_{3}}, \ldots, k_{s}^{m_{s}}\right)=J\left(k_{2}^{m_{2}}, k_{3}^{m_{3}}, \ldots, k_{s}^{m_{s}}\right) \\
+\left(p_{m_{1}+1}\left(k_{1}-k_{2}\right)-1\right) J\left(k_{2}^{m_{2}}, k_{3}^{m_{3}}, \ldots, k_{s}^{m_{s}}\right) \\
\quad+\sum_{j=2}^{\min \left\{m_{2}, k_{1}-k_{2}-1\right\}}
\end{gathered}
$$

Now, by Corollary $4.2, J\left(k_{2}^{m_{2}-j}, k_{3}^{m_{3}}, \ldots, k_{s}^{m_{s}}\right) \leqslant J\left(k_{2}^{m_{2}}, k_{3}^{m_{3}}, \ldots, k_{s}^{m_{s}}\right)$ for any $j \leqslant m_{2}$. It follows from the formula for $J(\lambda)$ in Theorem 4.1 that we have

$$
J(\lambda) \leqslant J\left(\left(k_{1}-k_{2}+1\right)^{m_{1}}, 1^{m_{2}}\right) J\left(k_{2}^{m_{2}}, k_{3}^{m_{3}}, \ldots, k_{s}^{m_{s}}\right) .
$$


In the case $k_{1}-k_{2}=1$, then equality follows since by Corollary $4.1, J\left(2^{m_{1}}, 1^{m_{2}}\right)=1$ and $J\left(k_{1}^{m_{1}}, k_{2}^{m_{2}}, \ldots, k_{s}^{m_{s}}\right)=J\left(k_{2}^{m_{2}}, k_{3}^{m_{3}}, \ldots, k_{s}^{m_{s}}\right)$.

Now suppose $m_{2} \geqslant k_{1}-k_{3}-2$. Then for $0 \leqslant j \leqslant k_{1}-k_{2}-1$ and $j \leqslant m_{2}$, we have $m_{2} \geqslant m_{2}-j \geqslant k_{2}-k_{3}-1$. Then by Corollary 4.1 ,

$$
J\left(k_{2}^{m_{2}}, k_{3}^{m_{3}}, \ldots, k_{s}^{m_{s}}\right)=J\left(k_{2}^{m_{2}-j}, k_{3}^{m_{3}}, \ldots, k_{s}^{m_{s}}\right)=J\left(k_{2}^{k_{2}-k_{3}-1}, k_{3}^{m_{3}}, \ldots, k_{s}^{m_{s}}\right) .
$$

By (5.1) and Theorem 4.1, we obtain $J(\lambda)=J\left(\left(k_{1}-k_{2}+1\right)^{m_{1}}, 1^{m_{2}}\right) J\left(k_{2}^{m_{2}}, k_{3}^{m_{3}}, \ldots, k_{s}^{m_{s}}\right)$, as claimed.

By induction, suppose the statements hold for all indices up to some $i \geqslant 2$ for any partition. By Theorem 4.1, we have

$$
\begin{aligned}
J\left(\left(k_{1}-k_{i+1}+1\right)^{m_{1}},\left(k_{2}-k_{i+1}+1\right)^{m_{2}}, \ldots, 1^{m_{i+1}}\right)=J\left(\left(k_{2}-k_{i+1}+1\right)^{m_{2}}, \ldots, 1^{m_{i+1}}\right) \\
+\left(p_{m_{1}+1}\left(k_{1}-k_{2}\right)-1\right) J\left(\left(k_{2}-k_{i+1}+1\right)^{m_{2}-1}, \ldots, 1^{m_{i+1}}\right) \\
+\sum_{j=2}^{\min \left\{m_{2}, k_{1}-k_{2}-1\right\}} p_{j+1}\left(k_{1}-k_{2}-1-j\right) J\left(\left(k_{2}-k_{i+1}+1\right)^{m_{2}-j}, \ldots, 1^{m_{i+1}}\right) .
\end{aligned}
$$

By the induction hypothesis, we have, for any $j$ such that $0 \leqslant j \leqslant m_{2}$,

$$
J\left(k_{2}^{m_{2}-j}, k_{3}^{m_{3}}, \ldots, k_{s}^{m_{s}}\right) \geqslant J\left(\left(k_{2}-k_{i+1}+1\right)^{m_{2}-j},\left(k_{3}-k_{i+1}+1\right)^{m_{3}}, \ldots, 1^{m_{i+1}}\right),
$$

and it follows from the expression for $J(\lambda)$ in Theorem 4.1 that

$$
J(\lambda) \geqslant J\left(\left(k_{1}-k_{i+1}+1\right)^{m_{1}},\left(k_{2}-k_{i+1}+1\right)^{m_{2}}, \ldots, 1^{m_{i+1}}\right) .
$$

This completes the induction argument for the first inequality.

We also have, by our induction hypothesis for the other two claims, that for any $0 \leqslant j \leqslant m_{2}$

$$
\begin{aligned}
J\left(k_{2}^{m_{2}-j}, k_{3}^{m_{3}}, \ldots, k_{s}^{m_{s}}\right) \leqslant J\left(\left(k_{2}-k_{i+1}+1\right)^{m_{2}-j},\left(k_{3}-k_{i+1}+1\right)^{m_{3}}, \ldots, 1^{m_{i+1}}\right) \\
\cdot J\left(k_{i+1}^{m_{i+1}}, \ldots, k_{s}^{m_{s}}\right),
\end{aligned}
$$

where equality holds when either $k_{i}-k_{i-1}=1$ or $m_{i+1} \geqslant k_{i}-k_{i+2}-2$. By applying this induction hypothesis and Theorem 4.1, we have

$$
\begin{aligned}
J(\lambda)= & J\left(k_{2}^{m_{2}}, \ldots, k_{s}^{m_{s}}\right)+\left(p_{m_{1}+1}\left(k_{1}-k_{2}\right)-1\right) J\left(k_{2}^{m_{2}-1}, k_{3}^{m_{3}}, \ldots, k_{s}^{m_{s}}\right) \\
& +\sum_{j=2}^{\min \left\{m_{2}, k_{1}-k_{2}-1\right\}} p_{j+1}\left(k_{1}-k_{2}-1-j\right) J\left(k_{2}^{m_{2}-j}, k_{3}^{m_{3}}, \ldots, k_{s}^{m_{s}}\right) \\
\leqslant & {\left[J\left(\left(k_{2}-k_{i+1}+1\right)^{m_{2}}, \ldots, 1^{m_{i+1}}\right)\right.} \\
& +\left(p_{m_{1}+1}\left(k_{1}-k_{2}\right)-1\right) J\left(\left(k_{2}-k_{i+1}+1\right)^{m_{2}-1}, \ldots, 1^{m_{i+1}}\right) \\
& \left.+\sum_{j=2}^{\min \left\{m_{2}, k_{1}-k_{2}-1\right\}} p_{j+1}\left(k_{1}-k_{2}-1-j\right) J\left(\left(k_{2}-k_{i+1}+1\right)^{m_{2}-j}, \ldots, 1^{m_{i+1}}\right)\right] \\
& \cdot J\left(k_{i+1}^{m_{i+1}}, \ldots, k_{s}^{m_{s}}\right)
\end{aligned}
$$


where equality holds if $k_{i}-k_{i-1}=1$ or $m_{i+1} \geqslant k_{i}-k_{i+2}-2$. Therefore, by (5.2), we have

$$
J(\lambda) \leqslant J\left(\left(k_{1}-k_{i+1}+1\right)^{m_{1}},\left(k_{2}-k_{i+1}+1\right)^{m_{2}}, \ldots, 1^{m_{i+1}}\right) J\left(k_{i+1}^{m_{i+1}}, \ldots, k_{s}^{m_{s}}\right),
$$

with equality when either $k_{i}-k_{i-1}=1$ or $m_{i+1} \geqslant k_{i}-k_{i+2}-2$, which completes the induction.

Example. Consider $\lambda=\left(18^{2}, 16,15^{2}, 12,8^{5}, 5\right)$. To compute $J(\lambda)$ using Theorem 4.1 would require several steps in the recursion. Instead, we apply Theorem 5.1, and working from smaller parts to larger parts of $\lambda$, we obtain

$$
\begin{aligned}
J(\lambda) & =J\left(11^{2}, 9,8^{2}, 5,1^{5}\right) J\left(8^{5}, 5\right) \\
& =J\left(4^{2}, 2,1^{2}\right) J\left(8^{2}, 5,1^{5}\right) J\left(8^{5}, 5\right) .
\end{aligned}
$$

It is now relatively quick to apply Theorem 4.1 and compute that $J\left(4^{2}, 2,1^{2}\right)=2$, $J\left(8^{2}, 5,1^{5}\right)=7$, and $J\left(8^{5}, 5\right)=9$, giving $J(\lambda)=126$.

We now use the inequality statements in Theorem 5.1 to obtain several results on classifying which $\lambda$ satisfy $J(\lambda)=n$ for small fixed $n$. First, we answer the question of which partitions $\lambda$ satisfy $K_{\lambda \mu}=1$ if and only if $\lambda=\mu$. While this result may be obtained by a combinatorial argument, we note that it follows almost immediately from the machinery that has been established.

Corollary 5.1. Let $\lambda=\left(k_{1}^{m_{1}}, \ldots, k_{s}^{m_{s}}\right)$. Then $J(\lambda)=1$ if and only if $k_{i-1}-k_{i}=k_{s}=1$ for all $i \geqslant 2$. Equivalently, $J(\lambda)=1$ if and only if $\lambda^{\prime}$ has distinct parts.

Proof. First, if $k_{i-1}-k_{i}=k_{s}=1$ for all $i \geqslant 2$, then it follows from Corollary 4.1 that $J(\lambda)=\left(1^{m_{s}}\right)=1$. Conversely, suppose that either $k_{s}>1$ or $k_{i-1}-k_{i}>1$ for some $2 \leqslant i \leqslant s$. If $k_{s}>1$, then by Corollary $4.2, J(\lambda) \geqslant J\left(k_{s}\right)=p\left(k_{s}\right)>1$. If $k_{i-1}-k_{i}>1$ for some $2 \leqslant i \leqslant s$, then by Theorem 5.1, followed by applying Corollary 4.2, we have

$$
J(\lambda) \geqslant J\left(\left(k_{1}-k_{i}+1\right)^{m_{1}}, \ldots,\left(k_{i-1}-k_{i}+1\right)^{m_{i-1}}, 1^{m_{i}}\right) \geqslant J\left(k_{i-1}-k_{i}+1,1^{m_{i}}\right) .
$$

By Theorem 4.1 , since $J\left(1^{m}\right)=1$ for any $m$, we have $J\left(k_{i-1}-k_{i}+1,1^{m_{i}}\right) \geqslant p_{2}\left(k_{i-1}-k_{i}\right) \geqslant$ 2 , since $k_{i-1}-k_{i} \geqslant 2$. Thus $J(\lambda)>1$.

Finally, it follows from the definition of $\lambda^{\prime}$ that the parts of $\lambda^{\prime}$ are distinct if and only if $k_{i-1}-k_{i}=k_{s}=1$ for all $i \geqslant 1$.

It is slightly more complicated to describe the set of $\lambda$ such that $J(\lambda)=2$, which we do now.

Corollary 5.2. Let $\lambda=\left(k_{1}^{m_{1}}, \ldots, k_{s}^{m_{s}}\right)$, and set $k_{s+1}=0$. Then $J(\lambda)=2$ if and only if one of the following conditions holds:

1. There exists a $j, 1 \leqslant j \leqslant s$, such that $k_{j}-k_{j+1}=2$, and $k_{i}-k_{i+1}=1$ for all $i \neq j$, $1 \leqslant i \leqslant s$. 
2. There exists a $j, 1 \leqslant j<s$, such that $k_{j}-k_{j+1}=3, m_{j}=m_{j+1}=1$, and $k_{i}-k_{i+1}=1$ for all $i \neq j, 1 \leqslant i \leqslant s$.

Proof. If either of the conditions holds, then by Corollary 4.1, we must have $J(\lambda)=$ $J\left(k_{j}^{m_{j}}, k_{j+1}^{m_{j+1}}, \ldots, k_{s}^{m_{s}}\right)$. Since $k_{j+1}-k_{j+2}=1$, then by Theorem 5.1 , we have

$$
\begin{aligned}
J(\lambda) & =J\left(\left(k_{j}-k_{j+1}+1\right)^{m_{j}}, 1^{m_{j+1}}\right) J\left(k_{j+1}^{m_{j+1}}, \ldots, k_{s}^{m_{s}}\right) \\
& =J\left(\left(k_{j}-k_{j+1}+1\right)^{m_{j}}, 1^{m_{j+1}}\right),
\end{aligned}
$$

where the second equality follows from Corollary 4.1 and the assumption that $k_{i}-k_{i+1}=1$ for $i \neq j$. Under the condition that $k_{j}-k_{j+1}=2$, we have $J(\lambda)=J\left(3^{m_{j}}, 1^{m_{j+1}}\right)$, and it follows from Theorem 4.1 that $J(\lambda)=2$. If $k_{j}-k_{j+1}=3$ and $m_{j}=m_{j+1}=1$, then $J(\lambda)=J(4,1)=2$.

Now suppose $J(\lambda)=2$. By Corollary 5.1, there must be at least one $j$ such that $k_{j}-k_{j+1}>1$. Suppose that, for some $j, k_{j}-k_{j+1} \geqslant 4$. Then, by Corollary 4.2 and Theorem $5.1, J(\lambda) \geqslant J\left(k_{j}-k_{j+1}+1,1^{m_{j+1}}\right)$. Then by Theorem 4.1, $J(\lambda) \geqslant p_{2}\left(k_{j}-k_{j+1}\right) \geqslant p_{2}(4)=3$. Next suppose that for some $j, k_{j}-k_{j+1}=3$ and either $m_{j}>1$ or $m_{j+1}>1$. Then $J(\lambda) \geqslant J\left(4^{m_{j}}, 1^{m_{j+1}}\right)$. By Theorem 4.1, if $m_{j}>1$, then $J\left(4^{m_{j}}, 1^{m_{j+1}}\right) \geqslant p_{m_{j}+1}(3)=3$. If $m_{j}=1$ and $m_{j+1}>1$, then $J\left(4,1^{m_{j+1}}\right)=p_{2}(3)+p_{3}(0)=3$. If $k_{s}=3$, then by Corollary $4.2, J(\lambda) \geqslant J(3)=p(3)=3$. Finally, suppose that there are two different indices, $j$ and $j^{\prime}$, such that $k_{j}-k_{j+1} \geqslant 2$ and $k_{j^{\prime}}-k_{j^{\prime}+1} \geqslant 2$, and we take $j^{\prime}$ to be minimal such that $j^{\prime}>j$ with this property. Thus, either $j^{\prime}=j+1$, or for all $i$ such that $j<i<j^{\prime}$, we have $k_{i}-k_{i+1}=1$. If $j^{\prime}=j+1$, we have, by Corollary 4.2 and Theorem 5.1,

$$
J(\lambda) \geqslant J\left(k_{j}-k_{j+2}+1,\left(k_{j+1}-k_{j+2}+1\right)^{m_{j+1}}, 1^{m_{j+2}}\right) .
$$

Since $k_{j+1}-k_{j+2} \geqslant 2$, then $J\left(\left(k_{j+1}-k_{j+2}+1\right)^{m_{j+1}}, 1^{m_{j+2}}\right) \geqslant 2$. By Theorem 4.1, then,

$$
J\left(k_{j}-k_{j+2}+1,\left(k_{j+1}-k_{j+2}+1\right)^{m_{j+1}}, 1^{m_{j+2}}\right) \geqslant 2+\left(p_{2}\left(k_{j}-k_{j+1}\right)-1\right) \geqslant 3,
$$

since $k_{j}-k_{j+1} \geqslant 2$. So $J(\lambda)>2$ in this case. If $j^{\prime}>j+1$, then there is an $i, j<i<j^{\prime}$, such that $k_{i}-k_{i+1}=1$. By Theorem 5.1,

$$
J(\lambda)=J\left(\left(k_{1}-k_{i+1}+1\right)^{m_{1}}, \ldots, 1^{m_{i+1}}\right) J\left(k_{i+1}^{m_{i+1}}, \ldots, k_{s}^{m_{s}}\right) .
$$

Since $\left(k_{j}-k_{i+1}+1\right)-\left(k_{j+1}-k_{i+1}+1\right)=k_{j}-k_{j+1} \geqslant 2$, then by Corollary 5.1, $J\left(\left(k_{1}-\right.\right.$ $\left.\left.k_{i+1}+1\right)^{m_{1}}, \ldots, 1^{m_{i+1}}\right) \geqslant 2$. Since $k_{j^{\prime}}-k_{j^{\prime}+1} \geqslant 2$, then $J\left(k_{i+1}^{m_{i+1}}, \ldots, k_{s}^{m_{s}}\right) \geqslant 2$, again by Corollary 5.1. Thus $J(\lambda) \geqslant 4$ in this case. Therefore, if neither conditions 1 nor 2 hold, then $J(\lambda) \neq 2$.

We could, with more effort, attempt to classify $\lambda$ such that $J(\lambda)=3, J(\lambda)=4$, and so on, undoubtedly with longer and longer lists of conditions. Instead, we prove the following general result in this direction.

Corollary 5.3. Suppose $\lambda=\left(k_{1}^{m_{1}}, \ldots, k_{s}^{m_{s}}\right)$ and that $J(\lambda)$ is prime. Then there exist $j$ and $j^{\prime}, j \leqslant j^{\prime}$, such that, for all $i$ such that $0<i<j$ or $j^{\prime}<i<s+1, k_{i}-k_{i+1}=1$, and for all $i$ such that $j \leqslant i \leqslant j^{\prime}, k_{i}-k_{i+1}>1$. 
Proof. Let $j$ be minimal such that $k_{j}-k_{j+1}>1$, and such a $j$ exists by Corollary 5.1. That is, $k_{i}-k_{i+1}=1$ for all $i<j$. If for all $i \geqslant j$, we have $k_{i}-k_{i+1}>1$, we are done by taking $j^{\prime}=s$. Otherwise let $j^{\prime}$ be minimal such that $j \leqslant j^{\prime}<s, k_{j^{\prime}}-k_{j^{\prime}+1}>1$, and $k_{j^{\prime}+1}-k_{j^{\prime}+2}=1$, so $k_{i}-k_{i+1}>1$ whenever $j \leqslant i \leqslant j^{\prime}$. Then by Theorem 5.1 , we have

$$
J(\lambda)=J\left(\left(k_{1}-k_{j^{\prime}+2}+1\right)^{m_{1}}, \ldots, 1^{m_{j^{\prime}+2}}\right) J\left(k_{j^{\prime}+2}^{m_{j^{\prime}+2}}, \ldots, k_{s}^{m_{s}}\right) .
$$

Since $\left(k_{j^{\prime}}-k_{j^{\prime}+2}+1\right)-\left(k_{j^{\prime}+1}-k_{j^{\prime}+2}+1\right)=k_{j^{\prime}}-k_{j^{\prime}+1}>1$, then by Corollary $5.1, J\left(\left(k_{1}-\right.\right.$ $\left.\left.k_{j^{\prime}+2}+1\right)^{m_{1}}, \ldots, 1^{m_{j^{\prime}+2}}\right)>1$. Since $J(\lambda)$ is prime, we must have $J(\lambda)=J\left(\left(k_{1}-k_{j^{\prime}+2}+\right.\right.$ $\left.1)^{m_{1}}, \ldots, 1^{m_{j^{\prime}+2}}\right)$ and $J\left(k_{j^{\prime}+2}^{m_{j^{\prime}+2}}, \ldots, k_{s}^{m_{s}}\right)=1$. By Corollary 5.1, and since $k_{j^{\prime}+1}-k_{j^{\prime}+2}=1$, we must have $k_{i}-k_{i+1}=1$ for all $i>j^{\prime}$.

\section{Signed tableaux}

Augment the non-negative integers by symbols $\bar{i}$, for each positive integer $i$, such that $i-1<\bar{i}<i$, so

$$
0<\overline{1}<1<\overline{2}<2<\overline{3}<3<\cdots .
$$

Given a partition $\lambda$, a signed Young tableau of shape $\lambda$ is a filling of the Young diagram for $\lambda$ with elements from the set $\{0, \overline{1}, 1, \overline{2}, 2, \overline{3}, 3, \ldots\}$, with rows non-decreasing from left to right, and columns increasing from top to bottom, with respect to the order just defined. We shall refer to these as simply signed tableaux from now on. The weight of a signed tableau $T$ is a tuple wt $(T)=\left(w_{0}, w_{1}, w_{2}, \ldots\right)$, where $w_{0}$ is the number of 0 's in $T$, and for $i>0, w_{i}$ is the number of $i$ 's plus the number of $\bar{i}$ 's in $T$.

For example, if $\lambda=(5,4,3,3,1)$, then

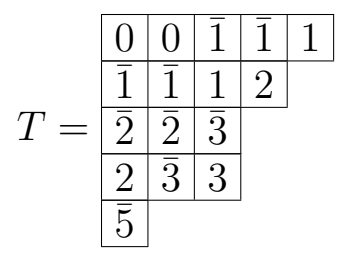

is a signed tableaux of shape $\lambda$ and weight $\operatorname{wt}(T)=(2,6,4,3,0,1)$.

Given a partition $\lambda$ and a tuple $\underline{w}=\left(w_{0}, w_{1}, w_{2}, \ldots\right)$, define the signed Kostka number $K_{\lambda, \underline{w}}^{ \pm}$to be the number of signed tableaux of shape $\lambda$ and weight $\underline{w}$. The signed Kostka numbers appear naturally in symmetric function theory, like their unsigned counterpart, in the following way. For any positive integer $i$, let $h_{i}$ denote the complete symmetric function of degree $i$, and for any partition $\lambda$ of $n$, let $s_{\lambda}$ be the Schur symmetric function. Let $\mathcal{P}_{n}$ denote the set of all partitions of $n$. Then, for any positive integer $n$, and any tuple $\left(w_{0}, w_{1}, \ldots, w_{\ell}\right)$ such that $\sum_{j=0}^{\ell} w_{j}=n$, as shown in [5, Lemma 4.2(a)], we have

$$
h_{w_{0}} \prod_{j=0}^{\ell} \sum_{i=0}^{w_{j}} h_{i} h_{w_{j}-i}=\sum_{\lambda \in \mathcal{P}_{n}}\left(K_{\lambda,\left(w_{0}, w_{1}, \ldots, w_{\ell}\right)}^{ \pm}\right) s_{\lambda} .
$$


It follows immediately from (6.1) that the signed Kostka number $K_{\lambda,\left(w_{0}, w_{1}, \ldots, w_{\ell}\right)}^{ \pm}$is invariant under both inserting or removing 0 's from $\left(w_{1}, \ldots, w_{\ell}\right)$ and under permutation action on $\left(w_{1}, \ldots, w_{\ell}\right)$. When studying the signed Kostka number, then, we often consider weights $\underline{w}=\left(w_{0}, \mu\right)$ such that $\mu$ is a partition.

Signed tableaux come up naturally when computing multiplicities of characters in degenerate Gelfand-Graev characters of finite unitary groups [5], and in this context the weight $\left(w_{0}, w_{1}, w_{2}, \ldots\right)$ also has the property that $\mu=\left(w_{1}, w_{2}, \ldots\right)$ is a partition. We thus address the question: Given a partition $\lambda$, for what non-negative integer $w_{0}$ and partition $\mu$ (with $|\lambda|=w_{0}+|\mu|$ ) is it true that $K_{\lambda,\left(w_{0}, \mu\right)}^{ \pm}=1$ ? The next result tells us that there are quite strong restrictions on $\lambda$ for there to even exist a weight $\underline{w}$ such that $K_{\lambda, \underline{w}}^{ \pm}=1$. We note that the main idea of the following result is very similar to [5, Proposition 5.1] and its proof.

Proposition 6.1. Given a partition $\lambda$ and a tuple $\underline{w}=\left(w_{0}, w_{1}, w_{2}, \ldots\right)$, suppose that $K_{\lambda, \underline{w}}^{ \pm}=1$, so $|\lambda|=\sum_{i} w_{i}$, and let $T$ be the unique signed tableau of shape $\lambda$ and weight $\underline{w}$. Then, $\lambda$ has at most one part with odd multiplicity, and if such a part exists it must have size $w_{0}$, otherwise $w_{0}=0$. Furthermore, for every positive integer $i$, any $i$ occurring in $T$ must be directly below some $\bar{i}$, and any $\bar{i}$ in $T$ must be directly above some $i$. That is, if we remove the first $w_{0}$ boxes from the first row of $T$ (which we denote $\left.T /\left(w_{0}\right)\right)$, the rest of the tableau is tiled with vertical dominoes of the form $\frac{\bar{i}}{i}$.

Proof. We first explain the last statement, as it implies the other statements. Suppose that in the tableau $T$, there is some $\bar{i}$ with no $i$ below it. Taking the $\bar{i}$ furthest to the right in the same row, we may change that $\bar{i}$ to an $i$ to obtain a second signed tableau of the same shape and weight. Similarly, if there is some $i$ with no $\bar{i}$ above it, then consider the $i$ furthest to the left in the same row, and we may change it to an $\bar{i}$, contradicting the assumption that $K_{\lambda, \underline{w}}^{ \pm}=1$.

Now suppose $\lambda$ has no parts with odd multiplicity, which means every column of $\lambda$ has even length. If $w_{0} \geqslant 1$, then the first column of $T /\left(w_{0}\right)$ may not be tiled by vertical dominoes, a contradiction. Thus $w_{0}=0$ in this case. Now suppose $\lambda$ has parts with odd multiplicity, and that $y$ is the largest part with odd multiplicity. We show $w_{0}=y$. If $w_{0}<y$, then the $y$ th column of $\lambda$ has odd length, and $T /\left(w_{0}\right)$ cannot be tiled with vertical dominoes, a contradiction. If $w_{0}>y$, then the $w_{0}$-th column of $T /\left(w_{0}\right)$ has odd length, and cannot be tiled with vertical dominoes. Thus $w_{0}=y$. Finally, suppose that $\lambda$ has another part with odd multiplicity, say of size $x$, where we then must have $x<y$. This implies the $x$ th column of $\lambda$ has even length. Then $x<w_{0}=y$, and the $x$ th column of $T /\left(w_{0}\right)$ may not be tiled by vertical dominoes. Thus $\lambda$ has at most one part with odd multiplicity, which has size $w_{0}$ if it exists, and $w_{0}$ otherwise.

Similar to the previous sections, if we define $J^{ \pm}(\lambda)$ to be the number of weights $\underline{w}$ such that $K_{\lambda, \underline{w}}^{ \pm}=1$, then Proposition 6.1 says that for any partition $\lambda, J^{ \pm}(\lambda)=0$ unless $\lambda$ has at most one part with odd multiplicity. In fact, in the case that $\lambda$ has at most one part with odd multiplicity, we will be able to say there is a unique weight $\underline{w}=\left(w_{o}, \mu\right)$ 
such that $\mu$ is a partition with the property that $K_{\lambda,\left(w_{0}, \mu\right)}^{ \pm}=1$. Given a partition $\lambda$ with at most one part with odd multiplicity, define the maximal signed tableau of shape $\lambda$ as follows.

For each column of the Young diagram of $\lambda$ of odd length (that is, columns which intersect a part with odd multiplicity), say of length $2 l+1$, fill these boxes from top to bottom with the elements $0, \overline{1}, 1, \overline{2}, 2, \ldots, \bar{l}, l$. For each column of even length, say $2 h$, fill these boxes with the elements $\overline{1}, 1, \overline{2}, 2, \ldots, \bar{h}, h$. For example, if $\lambda=(6,6,4,4,4,3,3,1,1)$, the maximal signed tableaux of shape $\lambda$ is

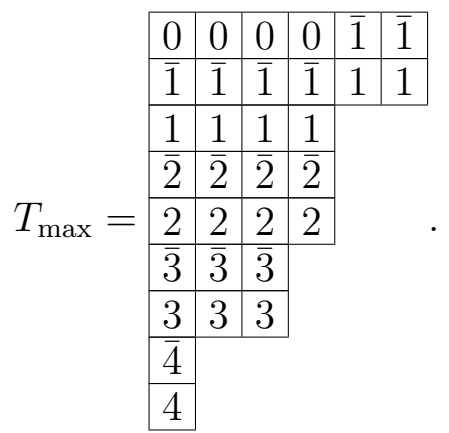

The maximal weight of such a $\lambda$ is the weight $\operatorname{wt}\left(T_{\max }\right)$ of the maximal signed tableau of shape $\lambda$. In the above example, the maximal weight is $(4,12,8,6,2)$. Specifically, let $\lambda=\left(\lambda_{1}, \lambda_{2}, \ldots, \lambda_{\ell}\right)=\left(k_{1}^{m_{1}}, k_{2}^{m_{2}}, \ldots, k_{j}^{m_{j}}, \ldots, k_{s}^{m_{s}}\right)$, where if $k_{j}$ is the unique part of $\lambda$ with odd multiplicity $m_{j}$, then set $w_{0}=k_{j}$, and if $\lambda$ has no part with odd multiplicity, set $w_{0}=0$ and assume $m_{j}$ is even. The maximal weight is then given by $\left(w_{0}, \mu\right)$, where $\mu$ is the partition

$$
\mu=\left(\left(2 k_{1}\right)^{m_{1} / 2},\left(2 k_{2}\right)^{m_{2} / 2}, \ldots,\left(2 k_{j}\right)^{\left\lfloor m_{j} / 2\right\rfloor}, \ldots,\left(2 k_{s}\right)^{m_{s} / 2}\right) .
$$

If $\left(w_{0}, \mu\right)$ is the maximal weight for a partition $\lambda$, note that $K_{\lambda,\left(w_{0}, \mu\right)}^{ \pm}=1$ by construction, since, given that $w_{0}$ is the number of 0's in a signed tableau of shape $\lambda$, then $\mu_{1}$ is the maximum number of $\overline{1}$ 's and 1's that can be in the tableau, and $\mu_{2}$ is the maximum number of $\overline{2}$ 's and 2's that can be in the tableau, and so on, so that the maximum tableau of shape $\lambda$ is the only such tableau of this shape and weight.

Next we show that the converse of this statement is true as well. That is, if $\lambda$ is a partition with at most one part with odd multiplicity, we will prove that $K_{\lambda, \underline{w}}^{ \pm}=1$ for some $\underline{w}=\left(w_{0}, \mu\right)$ with $\mu$ a partition if and only if $\underline{w}=\left(w_{0}, \mu\right)$ is the maximal weight for $\lambda$, and otherwise $K_{\lambda, w}^{ \pm}=0$. We will actually prove a stronger statement than this, by considering sequences of signed tableaux.

Let $\boldsymbol{\lambda}=\left(\lambda^{(j)}\right)=\left(\lambda^{(1)}, \lambda^{(2)}, \ldots, \lambda^{(n)}\right)$ be a finite sequence of partitions, or a $\mathrm{mul}$ tipartition. A finite sequence of signed tableaux, $\boldsymbol{T}=\left(T^{(j)}\right)=\left(T^{(1)}, T^{(2)}, \ldots, T^{(n)}\right)$ is said to have shape $\boldsymbol{\lambda}$ if $T^{(j)}$ has shape $\lambda^{(j)}$ for each $i$. Define the weight of $\boldsymbol{T}$ to be $\operatorname{wt}(\boldsymbol{T})=\sum_{j=1}^{n} \operatorname{wt}\left(T^{(j)}\right)$, where the sum of weights is taken component-wise. Given a finite sequence of partitions $\boldsymbol{\lambda}$, and a tuple $\underline{w}$, define $K_{\boldsymbol{\lambda}, w}^{ \pm}$to be the number of sequences of signed tableaux $\boldsymbol{T}$ of shape $\boldsymbol{\lambda}$ and weight $\underline{w}$.

Given a sequence of partitions $\boldsymbol{\lambda}$, by Proposition 6.1 , in order for there to exist a weight $\underline{w}$ such that $K_{\boldsymbol{\lambda}, \underline{w}}^{ \pm}=1$, it must be that for every $j, \lambda^{(j)}$ must have at most one part 
with odd multiplicity, and $w_{0}$ must be the sum of those part sizes with odd multiplicity. When $\boldsymbol{\lambda}$ satisfies this condition, define the maximal weight for the sequence $\boldsymbol{\lambda}$ to be the sum of the maximal weights of each $\lambda^{(j)}$ in the sequence. We may now prove the following uniqueness result.

Theorem 6.1. Let $\boldsymbol{\lambda}=\left(\lambda^{(j)}\right)$ be a sequence of partitions. Suppose $\underline{w}=\left(w_{0}, \mu\right)$ is such that $\mu$ is a partition, and such that $K_{\boldsymbol{\lambda},\left(w_{0}, \mu\right)}^{ \pm}=1$. Then each $\lambda^{(j)}$ has at most one part with odd multiplicity, $\left(w_{0}, \mu\right)$ is the maximal weight for $\boldsymbol{\lambda}$, and the unique sequence of signed tableaux $\boldsymbol{T}_{\max }=\left(T_{\max }^{(j)}\right)$ of shape $\boldsymbol{\lambda}$ and weight $\left(w_{0}, \mu\right)$ is the sequence such that each $T_{\max }^{(j)}$ is the maximal signed tableau of shape $\lambda^{(j)}$.

Proof. It follows directly from Proposition 6.1 that each $\lambda^{(j)}$ has at most one part with odd multiplicity, by considering each tableau in the sequence individually. So, suppose that $\boldsymbol{T}=\left(T^{(j)}\right)$ is a sequence of signed tableaux of shape $\boldsymbol{\lambda}$, such that $\operatorname{wt}(\boldsymbol{T})=\left(v_{0}, \nu\right)$ where $\nu$ is a partition, and suppose $\boldsymbol{T}$ is not the sequence $\boldsymbol{T}_{\max }$ of maximal signed tableaux. We must show that $K_{\boldsymbol{\lambda},\left(v_{0}, \nu\right)}^{ \pm} \neq 1$.

By Proposition 6.1, we may assume that for each $j$, the number of 0 's in $T^{(j)}$ is equal to the part size of $\lambda^{(j)}$ with odd multiplicity, and we may assume that in $T^{(j)}$, for any positive integer $i$, there is a $\bar{i}$ directly above any $i$, and an $i$ directly below any $\bar{i}$.

Since we are assuming $\boldsymbol{T}$ is not the sequence of maximal tableaux, let $r$ be the least positive integer such that $T^{(r)}$ is not the maximal tableau of shape $\lambda^{(r)}$. Consider the first (top-most) row of $T^{(r)}$ which differs from the maximal signed tableau, and consider the first (left-most) entry of that row at which this difference occurs. This entry must be of the form $\bar{i}$, since there must be an $\bar{i}$ above any $i$. By definition of this entry differing from the maximal signed tableau, then above this $\bar{i}$, there must be some non-negative integer $a$, where $a<i-1$, or this entry is in the first row, and $i>1$. In either case, in the tableau $T^{(r)}$, there is an entry $\bar{i}$, with $i>1$, and with no $i-1$ directly above it.

Now, we claim there must be a tableau $T^{\left(r^{\prime}\right)}$ in the sequence $\boldsymbol{T}$ which contains an entry $i-1$ with no $\bar{i}$ below it. Otherwise, since there is some $\bar{i}$ with no $i-1$ directly above it, then the total number of $\bar{i}$ 's would exceed the total number of $i-1$ 's in $\boldsymbol{T}$. However, we are assuming from Proposition 6.1 that the number of $i-1$ 's is equal to the number of $\overline{i-1}$ 's, and the number of $i$ 's is equal to the number of $\bar{i}$ 's, which would imply that in $\operatorname{wt}(\boldsymbol{T})=\left(v_{0}, \nu_{1}, \nu_{2}, \ldots\right)$, we have $\nu_{i}>\nu_{i-1}$, contradicting the assumption that $\nu$ is a partition.

We now have, in $T^{(r)}$, a left-most entry $\bar{i}$ with no $i-1$ directly above it, and in $T^{\left(r^{\prime}\right)}$, consider a right-most entry $i-1$ with no $\bar{i}$ directly below it. These two entries cannot be adjacent with $r=r^{\prime}$, since above the $i-1$ there must be a $\overline{i-1}$, while above the $\bar{i}$ there must be an entry strictly less than $\overline{i-1}$.

Now, exchange these two entries. We claim that this defines a sequence of signed tableaux, $\boldsymbol{S}=\left(S^{(j)}\right)$, where $S^{(j)}=T^{(j)}$, unless $j=r$ or $j=r^{\prime}$, in which case the entries just described have been exchanged. Consider first $S^{(r)}$, and the entry containing $i-1$ which previously contained $\bar{i}$. To the right of this entry is an entry $\bar{i}$ or greater, and below the entry is an $i$. Since this entry was chosen as the first difference in $T^{(r)}$ from the maximal tableau of shape $\lambda^{(r)}$, then above it must be an entry less than $i-1$, and to its 
left an entry less than $\bar{i}$. Thus, $S^{(r)}$ is a signed tableau. In the entry of $S^{\left(r^{\prime}\right)}$ containing $\bar{i}$ which previously contained $i-1$, there is no $\bar{i}$ below it, so the entry must be $\overline{i+1}$ or larger. To the right of this $\bar{i}$, there must be an entry $\bar{i}$ or larger, since if there was an $i-1$, it would have to have a $\bar{i}$ below it (since we chose the right-most $i-1$ with no $\bar{i}$ below it), which is impossible, since to the left of that entry is an entry $\overline{i+1}$ or larger. It follows that $S^{\left(r^{\prime}\right)}$ is a signed tableau. Thus, $\boldsymbol{S}$ is a second sequence of signed tableaux of shape $\boldsymbol{\lambda}$ and weight $\left(v_{0}, \nu\right)$, meaning $K_{\boldsymbol{\lambda},\left(v_{0}, \nu\right)}^{ \pm}>1$.

By applying Proposition 6.1 and Theorem 6.1 to a single partition, we have that $K_{\lambda,\left(w_{0}, \mu\right)}^{ \pm}=1$ if and only if $\lambda$ has at most one part with odd multiplicity and $\left(w_{0}, \mu\right)$ is the maximal weight for $\lambda$. This may rephrased as follows.

Corollary 6.1. For any partition $\lambda, J^{ \pm}(\lambda)=1$ if and only if $\lambda$ has at most one part with odd multiplicity, and otherwise $J^{ \pm}(\lambda)=0$.

For a sequence of more than one partition, the converse of Theorem 6.1 does not hold. For example, consider the pair of partitions $\lambda^{(1)}=(2,2,1), \lambda^{(2)}=(2,2,1,1)$, for which the maximum weight is $\left(w_{0}, \mu\right)=(1,6,2)$. However, the following is a sequence of tableaux of shape $\left(\lambda^{(1)}, \lambda^{(2)}\right)$ and weight $\left(w_{0}, \mu\right)$, but is not the sequence of maximal tableaux:

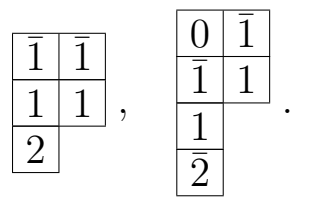

In the next result, we give a necessary and sufficient condition on a sequence of partitions $\boldsymbol{\lambda}$ for there to be a weight such that $K_{\boldsymbol{\lambda},\left(w_{0}, \mu\right)}^{ \pm}=1$, which we know must be unique by Theorem 6.1.

Theorem 6.2. Let $\boldsymbol{\lambda}=\left(\lambda^{(j)}\right)$ be a sequence of partitions, and $\left(w_{0}, \mu\right)$ be such that $\mu$ is a partition. Then $K_{\boldsymbol{\lambda},\left(w_{0}, \mu\right)}^{ \pm}=1$ if and only if every odd length column of every $\lambda^{(j)}$ is longer than every even length common of every $\lambda^{(j)}$, and $\left(w_{0}, \mu\right)$ is the maximal weight for $\boldsymbol{\lambda}$.

Proof. Suppose that $K_{\boldsymbol{\lambda},\left(w_{0}, \mu\right)}^{ \pm}=1$. By Theorem 6.1, we know that $\left(w_{0}, \mu\right)$ must be the maximal weight for $\boldsymbol{\lambda}$. Suppose that there is some odd length column, of $\lambda^{(j)}$, which is shorter than some even length column, of $\lambda^{\left(j^{\prime}\right)}$. Then we may assume $j \neq j^{\prime}$. Consider the right-most odd length column in $\lambda^{(j)}$, say of length $2 h+1$, and the left-most even length column in $\lambda^{\left(j^{\prime}\right)}$, say of length $2 l$, so $l>h$. In the maximal tableau of shape $\boldsymbol{\lambda}$, this odd length column has entries $0, \overline{1}, 1, \ldots, \bar{h}, h$, while the even length column has entries $\overline{1}, 1, \ldots, \bar{l}, l$. Replace the entries in the odd length column with the entries $\overline{1}, 1, \ldots, \bar{h}, h, l$, and the entries in the even length column with the entries $0, \overline{1}, 1, \ldots, \overline{h-1}, h-1, \bar{h}$. For example, suppose $h=2$ and $l=3$. Including the columns on either side of the columns 
of interest, this would look as follows:

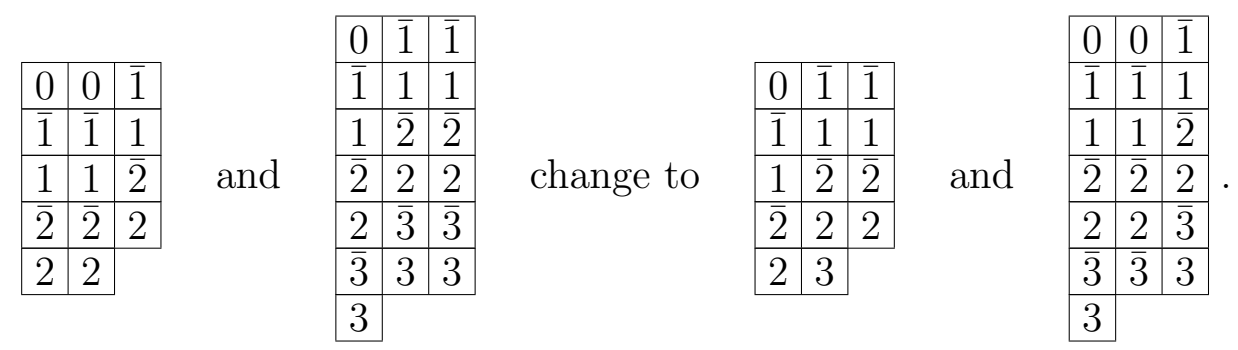

By our choice of columns, this defines a new sequence of signed tableaux of shape $\boldsymbol{\lambda}$ and weight $\left(w_{0}, \mu\right)$, and so $K_{\boldsymbol{\lambda},\left(w_{0}, \mu\right)}^{ \pm}>1$, a contradiction.

Conversely, suppose that everyone odd length column of every $\lambda^{(j)}$ is longer than every even length column of every $\lambda^{(j)}$. We must show that the only sequence of signed tableaux of shape $\boldsymbol{\lambda}$ and maximal weight is the sequence of maximal tableaux. By the definition of the maximum tableau $T_{\max }^{(j)}$ of shape $\lambda^{(j)}, T_{\max }^{(j)}$ contains the maximum number of $\overline{1}$ 's, 1 's, $\overline{2}$ 's,..., possible, given that the tableau contains the number of 0 's equal to the size of the part with odd multiplicity. Therefore, in order for there to be another sequence of tableaux of shape $\boldsymbol{\lambda}$ and weight $\left(w_{0}, \mu\right)$, one of the tableau in the sequence must have a different number of 0 than the number of odd length columns of that partitions, which is equal to the size of the part with odd multiplicity.

Consider all columns whose length is maximum among all $\lambda^{(j)}$. If this length is even, then there are no columns of odd length by assumption, and so $w_{0}=0$, and thus the sequence of maximal tableaux, by construction, is the only sequence of tableaux of shape $\boldsymbol{\lambda}$ and weight $\left(w_{0}, \mu\right)$. If this length is odd, say of length $2 h_{1}+1$, then the largest of any entry in the sequence of tableaux is $h_{1}$. In any of these longest columns, then, the entries from top to bottom must be $0, \overline{1}, 1, \ldots, \overline{h_{1}}, h_{1}$, since these are the only entries used in any of the tableaux. If the next longest column is of even length, then there are no other odd length columns, and so all 0's must be in the same positions as they are in the sequence of maximal tableaux, and we are done. Otherwise, if the next longest column is of odd length $2 h_{2}+1$, then the largest entry in any column of this length or shorter is $h_{2}$, and there is no choice but for all columns of this length to have entries $0, \overline{1}, 1, \ldots, \overline{h_{2}}, h_{2}$. Again, if there are no other odd length columns, then all 0's are in the same place as they are in the sequence of maximal tableaux. Otherwise, we continue in this way until no other odd length columns are left. Thus, when $\left(w_{0}, \mu\right)$ is the maximal weight for $\boldsymbol{\lambda}$, we have $K_{\lambda,\left(w_{0}, \mu\right)}^{ \pm}=1$.

We conclude by giving an application to the representations of the finite unitary group, following [5]. Let $\mathbb{F}_{q}$ be a finite field with $q$ elements, $\overline{\mathbb{F}}_{q}$ an algebraic closure, and define the map $F$ on $\overline{\mathbb{F}}_{q}^{\times}$by $F(a)=a^{-q}$. Let $\Phi$ be the set of $F$-orbits on $\overline{\mathbb{F}}_{q}^{\times}$, and let $\Theta$ be a certain set of $F$-orbits which are dual to $\Phi$, for a precise definition see [5, Section 2.4]. Let $\mathrm{U}\left(n, \mathbb{F}_{q}\right)$ denote the unitary group defined over $\mathbb{F}_{q}$. The irreducible complex characters of $\mathrm{U}\left(n, \mathbb{F}_{q}\right)$ are parameterized by functions $\boldsymbol{\lambda}: \Theta \rightarrow \mathcal{P}$, where $\mathcal{P}$ is the set of all partitions, such that $\sum_{\varphi \in \Theta}|\varphi||\boldsymbol{\lambda}(\varphi)|=n$ (see [5, Section 2.5]). Such $\boldsymbol{\lambda}$ are called $\Theta$-multipartitions 
of $n$. Let $\chi^{\boldsymbol{\lambda}}$ denote the complex irreducible character of $\mathrm{U}\left(n, \mathbb{F}_{q}\right)$ corresponding to the $\Theta$-multipartition $\boldsymbol{\lambda}$.

The degenerate Gelfand-Graev characters of $\mathrm{U}\left(n, \mathbb{F}_{q}\right)$ are representations obtained by induced one-dimensional characters from the Sylow $p$-subgroup of $\mathrm{U}\left(n, \mathbb{F}_{q}\right)$, where $p=$ $\operatorname{char}\left(\mathbb{F}_{q}\right)$. The distinct degenerate Gelfand-Graev characters of $\mathrm{U}\left(n, \mathbb{F}_{q}\right)$ are parameterized by pairs $(k, \nu)$, where $k$ is a positive integer, and $\nu$ is a partition, such that $k+2|\nu|=n$, and the degenerate Gelfand-Graev character corresponding to $(k, \nu)$ is denoted $\Gamma_{(k, \nu)}$ (see [5, Section 4.2]).

In [5, Theorem 4.4], it is shown that the multiplicity of $\chi^{\boldsymbol{\lambda}}$ in $\Gamma_{(k, \nu)}$ is another variation of the Kostka number, namely, the number of battery tableaux of shape $\boldsymbol{\lambda}$ and weight $(k, \nu)$ (see [5, Section 4.3]). In particular, a battery tableau is a certain sequence of tableaux indexed by $\Theta$, such that when $\varphi \in \Theta$ and $|\varphi|$ is even, then $\boldsymbol{\lambda}(\varphi)$ is a signed tableau. The following result gives a specific set of characters of $\mathrm{U}\left(n, \mathbb{F}_{q}\right)$ which appear with multiplicity 1 in exactly one degenerate Gelfand-Graev character.

Corollary 6.2. Let $\boldsymbol{\lambda}$ be a $\Theta$-partition of $n$, and let $\Theta_{\boldsymbol{\lambda}}$ be the support of $\boldsymbol{\lambda}$, that is, the set of all $\varphi \in \Theta$ such that $\boldsymbol{\lambda}(\varphi)$ is not the empty partition. Suppose there is an even integer $m$ such that $|\varphi|=m$ for every $\varphi \in \Theta_{\boldsymbol{\lambda}}$. Then $\chi^{\boldsymbol{\lambda}}$ appears with multiplicity 1 in $\Gamma_{(k, \nu)}$ if and only if every odd length column of every $\boldsymbol{\lambda}(\varphi)$ is longer than every even length column of every $\boldsymbol{\lambda}(\varphi), \varphi \in \Theta_{\boldsymbol{\lambda}}$, and $(k, \nu)$ is $\mathrm{m} / 2$ times the maximal weight for the sequence of partitions $(\boldsymbol{\lambda}(\varphi))_{\varphi \in \Theta_{\boldsymbol{\lambda}}}$.

Proof. This follows immediately from Theorems 6.1 and 6.2, and [5, Theorem 4.4].

Note that Corollary 6.2 explains the second example in the concluding remarks of [5].

Acknowledgements: The authors thank the editor Bruce Sagan, and the anonymous referee for suggestions to improve the paper.

\section{References}

[1] A. D. Berenshtein and A. V. Zelevinskii. When is the multiplicity of a weight equal to 1? Funct. Anal. Appl., 24(2):259-269, 1990.

[2] W. Fulton and J. Harris. Representation theory, a first course. Graduate Texts in Mathematics, 129. Springer-Verlag, New York, 1991.

[3] R. C. King. Weight multiplicities for the classical groups. In Group theoretical methods in physics (Fourth Internat. Colloq., Nijmegen, 1975), volume 50 of Lecture Notes in Phys., pages 490-499. Springer, Berlin, 1976.

[4] I. G. Macdonald. Symmetric functions and Hall polynomials, second edition, with contributions by A. Zelevinsky. Oxford Mathematics Monographs. Oxford Science Publications, The Clarendon Press, Oxford University Press, New York, 1995.

[5] N. Thiem and C. R. Vinroot. Gelfand-Graev characters of the finite unitary groups. Electron. J. Combin. 16(1):Research Paper 146, 37 pp, 2009. 